\title{
Displacement approach with external variables only for multi-domain analysis via symmetric BEM
}

\author{
L. Zito, T. Panzeca*, S. Terravecchia \\ Dipartimento di Ingegneria Strutturale, Aerospaziale e Geotecnica, Università di Palermo, Viale delle Scienze, 90128 Palermo, Italy
}

\section{A R T I C L E I N F O}

\section{Article history:}

Received 6 May 2009

Accepted 30 October 2010

Available online 28 December 2010

\section{Keywords:}

SBEM

Multi-domain analysis

Displacement approach

Closed form coefficients

\begin{abstract}
A B S T R A C T
In the present paper a new displacement method, defined as external variables one, is proposed inside the multidomain symmetric Boundary Element formulation.

This method is a natural evolution of the displacement approach with interface variables in the multidomain symmetric BEM analysis. Indeed, the strategy employed has the advantage of considering only the kinematical quantities of the free boundary nodes and the algebraic operators involved show symmetry and very small dimensions. The proposed approach is characterized by strong condensation of the mechanical and kinematical boundary nodes variables of the macro-elements. All the domain quantities, such as tractions and stresses, displacements and strains, are computed through the Somigliana Identities in a subsequent phase.

Some examples are shown using the calculus code Karnak.sGbem, by which it was possible to make some comparisons with analytical solutions and other approaches to show the effectiveness of the method proposed.
\end{abstract} (C) 2010 Elsevier Masson SAS. All rights reserved.

\section{Introduction}

In all fields of applied engineering there is the need to use analysis methods able to obtain solutions very close to the real ones through a reduced number of variables.

The Symmetric Boundary Element Method (SBEM), as well as the Collocation Boundary Element Method (BEM), has the peculiarity of working with variables of the boundary only. Furthermore, these methods utilize the Fundamental Solutions (F.S.) which allow one to obtain a high response accuracy because the equilibrium and compatibility equations are satisfied in the domain.

The computational difficulties related to the evaluation of some solving equation system coefficients, due to integrals with strong singularity or hypersingularity, initially slowed down application of the method. Later these difficulties were overcome by using different techniques: within the BEM, we can mention the papers by Gray et al. (1990), Portela et al. (1992), Holzer (1993), Guiggiani (1995), Aliabadi (2002) and, within the SBEM, the papers by Bonnet (1995), Frangi and Novati (1996), Panzeca et al. (1998), Salvadori (2001), Terravecchia (2006).

Other difficulties have been met in the analysis of the continuum bodies with step-wise variable physical and geometrical characteristics, but recently these drawbacks have been overcome through

\footnotetext{
* Corresponding author. Tel.: +39 91 6568413, fax: +39 916568407.

E-mail address: tpanzeca@tiscali.it (T. Panzeca).
}

appropriate strategies inside the substructuring process. The idea of subdividing the domain in the macro-elements was introduced, in the SBEM, by Maier et al. (1991). Subsequently Gray and Paulino (1997) utilized the substructuring in potential problems; Layton et al. (1997) proposed a formulation dividing the body in macroelements, each of which is governed by boundary quantities only, thus obtaining inside a condensation process a system having some non-symmetric blocks; Ganguly et al. (1999) presented an entirely symmetric approach for a plane elastic body subdivided by two macroelements, discretized along the boundary and characterized by a symmetric solving system having as unknowns all the boundary quantities, both those of the free and constrained boundaries and the interface one between the two macroelements.

Panzeca et al. dealt with the same problem by using a strategy connected to the variational formulation introduced by Maier and Polizzotto (1987), Polizzotto (1988a,b, 1991), and obtained two different approaches defined as mixed-variable (Panzeca et al., 1999; Panzeca and Salerno, 2000) and displacement approaches (Panzeca et al., 2002b,c), both characterized by a strong variable condensation. Through these strategies the authors obtained a system only depending on the interface nodal variables of the contiguous macroelements, here called bem-element (bem-e).

The approach, defined as displacement method with interface variables only, is very similar to that obtained by the FEM. The regularity conditions were imposed in weighted form for the tractions on the bem-e interface boundaries and for the displacements of the same boundary in each node. 
Subsequently, this formulation was applied by the same research group in some mechanics problems as the thermoelasticity (Panzeca et al., 2004), the contact-detachment problem (Panzeca et al., 2008), and the mechanics of quasi-brittle fracture (Panzeca et al., 2009).

Cen et al. (1999) developed a multi-zone approach, where a nonsymmetric mixed-variable solving system, having the unknown vector only with interface nodal mechanical and kinematical quantities, was considered within the cohesive fracture case.

Pérez-Gavilán and Aliabadi (2001) proposed an approach only having kinematical variables applied in problems having nonuniqueness solutions.

Kallivokas et al. (2005) proposed a variational formulation for multidomain interface problems in terms of Dirichlet-type variables only. The formulation was applied to thermal and acoustic problems.

Vodička et al. (2007) utilized a variational approach characterized by symmetric operators applied to nonconforming mesh problems for curve interfaces.

In the last decade several strategies with variables condensation have been developed allowing a reduction in variables, giving computationally more advantageous systems.

In this paper a strategy, defined as displacement approach with external variables only, is proposed. On the basis of an analogous strategy used by Panzeca et al. (2002b,c) within the displacement approach with inner variables, it leads to a solving equation system characterized by symmetric algebraic operators in which the unknowns are represented by the displacements of the free boundary nodes of the body.

The strategy contemplates:

- subdivision of the body into bem-es, having any dimension and shape;

- evaluation for each bem-e, discretized on the boundary, of an expression which relates the tractions evaluated in weighted (or generalized) form in the free or interface boundaries to the nodal displacements defined in the same boundaries and to the known domain and boundary actions;

- a solving equation system, obtained by imposing the equilibrium between the generalized tractions on the bem-e interface and the compatibility between the nodal displacements of the same boundaries, giving a system depending on all the interface boundary displacements and the free boundary displacements.

- reduction of the solving system unknowns through a condensation process, thus making it possible to have as unknowns the free boundary displacements only.

The approach works using as its only variables the free boundary displacements and leads to a solving system having very small dimensions. It does not involve changes in the solving system dimension when a new mesh is introduced in the domain or a new discretization is performed in the interface boundaries of the contiguous bem-es.

The method proposed was implemented within the Karnak.sGbem (Panzeca et al., 2002a) calculus code. This algorithm allows one to perform an elastic analysis for plates having any shape, made up of homogeneous material and having constant thickness for each bem-e, subjected to boundary forces, to imposed displacements, and to domain actions like body forces and inelastic strains, both transferred on the boundary of the bem-e according to a strategy developed by Panzeca et al. (2004).

Furthermore, the closed form evaluation of the matrix coefficients and of the load vector was carried out within the Karnak.sGbem calculus code according to a strategy shown subsequently by Terravecchia (2006). This strategy regards all the coefficients, both those concerning the boundary elements where cause distribution and effect evaluation are coincident and those distant from one another. Moreover, the evaluation of these coefficients, all in closed form, allows analysis of bem-es having any shape and dimension without giving rise to numerical instability.

\section{Multidomain elastic analysis}

Let us consider a bi-dimensional body having domain $\Omega$ and boundary $\Gamma$, referred to a Cartesian system $(0, x, y)$, subjected to actions in its plane:

- forces $\overline{\mathbf{f}}$ in the portion $\Gamma_{2}$ of the free boundary,

- displacements $\overline{\mathbf{u}}$ imposed in the portion $\Gamma_{1}$ of the constrained boundary,

- body forces $\overline{\mathbf{b}}$ and inelastic actions $\overline{\boldsymbol{\vartheta}}$ in $\Omega$.

In this body the physical and geometrical characteristics are zone-wise variables. We want to obtain the elastic response to the external actions in terms of displacements $\mathbf{u}_{2}$ on $\Gamma_{2}$, reactive forces $\mathbf{f}_{1}$ on $\Gamma_{1}$, displacements, strains and stresses in $\Omega$ by using the SBEM, through two formulations in the sphere of the displacement approach. For this purpose an appropriate subdivision of the domain into boundary elements (bem-es), each having constant physical and geometrical characteristics, was introduced.

This subdivision involves the introduction of an interface boundary $\Gamma_{0}$ between contiguous bem-es and, as a consequence, the rise of two new unknown quantities in the analysis problem, i.e. the displacements $\mathbf{u}_{0}$ and the interface tractions $\mathbf{f}_{0}$ vectors, both referred to interface boundaries.

Let us consider the i-th bem-e embedded in the infinite domain $\Omega_{\infty}$ having the same physical and geometrical characteristics as the element considered (Fig. 2). The boundary quantities of the bem-e are the forces $\mathbf{f}$ and the displacement discontinuities $(-\mathbf{u})$, both layered along the boundary.

The strategy adopted makes it possible to characterize each bem-e in a similar way to what is done in the FEM, but with high performances because of the peculiarity of the method employed; further, the closed form computation of the algebraic operator coefficients allows one to operate in a simple way in the presence of irregular bem-es.

Now two strategies are shown for performing multidomain analysis using the symmetric formulation of the BEM within the displacement approach. The first, shown in Section 3, is a classic displacement approach with interface variables, developed by Panzeca and Salerno (2000) and Panzeca et al. (2002b,c) where the substructuring problem is governed only by the kinematical nodal unknowns $\mathbf{U}_{0}$ of all the boundaries $\Gamma_{0}$. The second one, shown in Section 4, is a displacement approach with external variables, where the substructuring problem is governed only by the kinematical unknowns $\mathbf{U}_{2}$ of all the nodes of the body external boundaries $\Gamma_{2}$.

\section{Displacement approach with interface unknowns}

In this Section the strategy developed by Panzeca and Salerno (2000) and Panzeca et al. (2002b,c) is shown in performing multidomain analysis via SBEM, within the displacement approach.

A strategy is presented having the general characteristic of producing for each bem-e all the algebraic operators characterizing its mechanical behaviour depending on the geometry and boundary type. In this connection, the following relation, defined as characteristic, is obtained for each bem-e (Panzeca et al., 2002b,c):

$\mathbf{P}_{0}=\mathbf{D}_{00} \mathbf{U}_{0}+\underbrace{\widehat{\mathbf{P}}_{\Gamma}(\overline{\mathbf{f}}, \overline{\mathbf{u}})+\widehat{\mathbf{P}}_{\Omega}(\overline{\mathbf{b}}, \overline{\boldsymbol{\vartheta}})}_{\widehat{\mathbf{P}}_{0}}$. 
This relation, containing quantities evaluated at the interface among bem-es, connects the generalized tractions $\mathbf{P}_{0}$ along the boundary side to the nodal displacements $\mathbf{U}_{0}$, belonging to the same boundary through the stiffness matrix $\mathbf{D}_{00}$, and to the known generalized tractions regarding the boundary $\widehat{\mathbf{P}}_{\Gamma}$ and domain $\widehat{\mathbf{P}}_{\Omega}$ imposed actions.

If we impose some appropriate regularity conditions on the interface boundaries, the following solving equation system is obtained:

$\mathbf{K}_{00} \mathbf{U}_{0}+\widehat{\mathbf{f}}_{0}=\mathbf{0}$

where the unknowns are the displacements of all the nodes belonging only to the interface boundaries, and the operators $\mathbf{K}_{00}$ and $\widehat{\mathbf{f}}_{0}$ are the symmetric stiffness matrix of the structure and the load vector, respectively.

In Section 3.1 the characteristic equation of each e-bem is obtained by utilizing the Galerkin weighting definition. Subsequently in Section 3.2 the solving equation system is obtained by imposing appropriate regularity conditions, weak for the mechanical quantities and strong for the kinematical ones, both at the interface between contiguous bem-es.

\subsection{The elasticity relation of each bem-e}

For each bem-e we want to determine an elasticity equation connecting the quantities associated with the interface zone by using a strategy based on the SBEM. This strategy contemplates the study of each bem-e embedded in an unlimited domain having the same physical properties and the same thickness as the examining body. It is necessary to distinguish the body boundary as the boundary $\Gamma$ of $\Omega$ or as the boundary $\Gamma^{+}$of the complementary domain $\Omega_{\infty} \mid \Omega$. As a consequence of this condition the boundary quantities take on a different meaning: the forces acting on the boundary must be interpreted as layered force distribution, whereas the displacements must be thought of as double layered displacement distribution.

The displacements and the tractions marked with the symbols $(\cdot)^{+}$and $(\cdot)^{-}$denote boundary quantities defined on $\Gamma^{+}$and $\Gamma^{-}$, whose values may be defined at points infinitely close to $T$ from the outside and inside, respectively. However, for simplicity, the quantities referring to the inner boundary $\Gamma^{-}$are indicated without superscript because they represent the true quantities of the problem being examined, when the solution is obtained.

In order to obtain an elasticity relation for each of the bodies A, $B$, $C$, we refer to a generic body, thus omitting the reference superscript $A, B, C$ in the equations to be obtained.

Let us impose the classical Dirichlet and Neumann conditions, i.e.

$\mathbf{u}_{1}=\overline{\mathbf{u}}_{1}$ on $\Gamma_{1}$

$\mathbf{t}_{2}=\overline{\mathbf{f}}_{2}$ on $\Gamma_{2}$

and evaluate the displacement $\mathbf{u}_{0}$ and traction $\mathbf{t}_{0}$ vectors on $\Gamma_{0}$, i.e. in the interface zone.

When we introduce the Somigliana Identities (S.I.) of the displacements and the tractions, the following boundary integral equations can be obtained

on $\Gamma_{1}$

$$
\begin{gathered}
\int_{\Gamma_{1}} \mathbf{G}_{\mathrm{uu}} \mathbf{f}_{1}+\oint_{\Gamma_{1}} \mathbf{G}_{\mathrm{ut}}\left(-\overline{\mathbf{u}}_{1}\right)+\frac{1}{2} \overline{\mathbf{u}}_{1}+\int_{\Gamma_{2}} \mathbf{G}_{\mathrm{uu}} \overline{\mathbf{f}}_{2}+\int_{\Gamma_{2}} \mathbf{G}_{\mathrm{ut}}\left(-\mathbf{u}_{2}\right) \\
\quad+\int_{\Gamma_{0}} \mathbf{G}_{\mathrm{uu}} \mathbf{f}_{0}+\int_{\Gamma_{0}} \mathbf{G}_{\mathrm{ut}}\left(-\mathbf{u}_{0}\right)+\int_{\Omega} \mathbf{G}_{\mathrm{uu}} \overline{\mathbf{b}}+\int_{\Omega} \mathbf{G}_{\mathrm{u} \sigma} \overline{\boldsymbol{\vartheta}}=\overline{\mathbf{u}}_{1}
\end{gathered}
$$

$$
\begin{gathered}
\int_{\Gamma_{1}} \mathbf{G}_{\mathrm{tu}} \mathbf{f}_{1}+\int_{\Gamma_{1}} \mathbf{G}_{\mathrm{tt}}\left(-\overline{\mathbf{u}}_{1}\right)+\oint_{\Gamma_{2}} \mathbf{G}_{\mathrm{tu}} \overline{\mathbf{f}}_{2}+\frac{1}{2} \overline{\mathbf{f}}_{2}+\int_{\Gamma_{2}} \mathbf{G}_{\mathrm{tt}}\left(-\mathbf{u}_{2}\right) \\
\quad+\int_{\Gamma_{0}} \mathbf{G}_{\mathrm{tu}} \mathbf{f}_{0}+\int_{\Gamma_{0}} \mathbf{G}_{\mathrm{tt}}\left(-\mathbf{u}_{0}\right)+\int_{\Omega} \mathbf{G}_{\mathrm{tu}} \overline{\mathbf{b}}+\int_{\Omega} \mathbf{G}_{\mathrm{t} \sigma} \overline{\boldsymbol{\vartheta}}=\overline{\mathbf{f}}_{2}
\end{gathered}
$$

on $\Gamma_{0}$

$$
\begin{aligned}
\mathbf{u}_{0} & =\int_{\Gamma_{1}} \mathbf{G}_{\mathrm{uu}} \mathbf{f}_{1}+\int_{\Gamma_{1}} \mathbf{G}_{\mathrm{ut}}\left(-\overline{\mathbf{u}}_{1}\right)+\int_{\Gamma_{2}} \mathbf{G}_{\mathrm{uu}} \overline{\mathbf{f}}_{2}+\int_{\Gamma_{2}} \mathbf{G}_{\mathrm{ut}}\left(-\mathbf{u}_{2}\right) \\
& +\int_{\Gamma_{0}} \mathbf{G}_{\mathrm{uu}} \mathbf{f}_{0}+\oint_{\Gamma_{0}} \mathbf{G}_{\mathrm{ut}}\left(-\mathbf{u}_{0}\right)+\frac{1}{2} \mathbf{u}_{0}+\int_{\Omega} \mathbf{G}_{\mathrm{uu}} \overline{\mathbf{b}}+\int_{\Omega} \mathbf{G}_{\mathrm{u} \sigma} \overline{\boldsymbol{\vartheta}}
\end{aligned}
$$

$$
\begin{aligned}
\mathbf{t}_{0} & =\int_{\Gamma_{1}} \mathbf{G}_{\mathrm{tu}} \mathbf{f}_{1}+\int_{\Gamma_{1}} \mathbf{G}_{\mathrm{tt}}\left(-\overline{\mathbf{u}}_{1}\right)+\int_{\Gamma_{2}} \mathbf{G}_{\mathrm{tu}} \overline{\mathbf{f}}_{2}+\int_{\Gamma_{2}} \mathbf{G}_{\mathrm{tt}}\left(-\mathbf{u}_{2}\right) \\
& +\oint_{\Gamma_{0}} \mathbf{G}_{\mathrm{tu}} \mathbf{f}_{0}+\frac{1}{2} \mathbf{f}_{0}+\int_{\Gamma_{0}} \mathbf{G}_{\mathrm{tt}}\left(-\mathbf{u}_{0}\right)+\int_{\Omega} \mathbf{G}_{\mathrm{tu}} \overline{\mathbf{b}}+\int_{\Omega} \mathbf{G}_{\mathrm{t} \sigma} \overline{\boldsymbol{\vartheta}}
\end{aligned}
$$

where the small circles appearing in the integrals define these as Cauchy Principal Values (CPV), whereas the terms in which $1 / 2$ is found are the corresponding free terms. In addition, when the solution is reached, the traction vector $\mathbf{t}_{0}$, obtained by Eq. ( $\left.4 \mathrm{~d}\right)$ and defined in the equilibrium equation $\mathbf{f}_{0}=-\mathbf{t}_{0}^{+}+\mathbf{t}_{0}$ on the boundary, is identical to the layered force vector $\mathbf{f}_{0}$ because on $\Gamma_{0}^{+}$ the traction vector must be $\mathbf{t}_{0}^{+}=\mathbf{0}$.

Let us modify Eqs. (4a)-(4c) by grouping all the terms together on the left side and leaving Eq. (4d) unchanged. We obtain an integral equations system where the Eqs. (4a)-(4c) may be interpreted as the response of the examining body on the boundaries $\Gamma_{1}^{+}, \Gamma_{2}^{+}, \Gamma_{0}^{+}$, respectively, whereas Eq. (4d) maintains the same traction meaning as evaluated on the interface boundary $\Gamma_{0}$. We obtain

$$
\begin{aligned}
& \text { on } \Gamma_{1}^{+} \\
& \int_{\Gamma_{1}} \mathbf{G}_{\mathrm{uu}} \mathbf{f}_{1}+\int_{\Gamma_{2}} \mathbf{G}_{\mathrm{ut}}\left(-\mathbf{u}_{2}\right)+\int_{\Gamma_{0}} \mathbf{G}_{\mathrm{uu}} \mathbf{f}_{0}+\int_{\Gamma_{0}} \mathbf{G}_{\mathrm{ut}}\left(-\mathbf{u}_{0}\right) \\
& +\oint_{\Gamma_{1}} \mathbf{G}_{\mathrm{ut}}\left(-\overline{\mathbf{u}}_{1}\right)-\frac{1}{2} \overline{\mathbf{u}}_{1}+\int_{\Gamma_{2}} \mathbf{G}_{\mathrm{uu}} \overline{\mathbf{f}}_{2}+\int_{\Omega} \mathbf{G}_{\mathrm{uu}} \overline{\mathbf{b}}+\int_{\Omega} \mathbf{G}_{\mathrm{u} \sigma} \overline{\boldsymbol{\vartheta}}=\mathbf{0} \\
& \text { on } \Gamma_{2}^{+} \\
& \int_{\Gamma_{1}} \mathbf{G}_{\mathrm{tu}} \mathbf{f}_{1}+\int_{\Gamma_{2}} \mathbf{G}_{\mathrm{tt}}\left(-\mathbf{u}_{2}\right)+\int_{\Gamma_{0}} \mathbf{G}_{\mathrm{tu}} \mathbf{f}_{0}+\int_{\Gamma_{0}} \mathbf{G}_{\mathrm{tt}}\left(-\mathbf{u}_{0}\right) \\
& +\int_{\Gamma_{1}} \mathbf{G}_{\mathrm{tt}}\left(-\overline{\mathbf{u}}_{1}\right)+\oint_{\Gamma_{2}} \mathbf{G}_{\mathrm{tu}} \overline{\mathbf{f}}_{2}-\frac{1}{2} \overline{\mathbf{f}}_{2}+\int_{\Omega} \mathbf{G}_{\mathrm{tu}} \overline{\mathbf{b}}+\int_{\Omega} \mathbf{G}_{\mathrm{t} \sigma} \overline{\boldsymbol{\vartheta}}=0
\end{aligned}
$$

$$
\begin{aligned}
& \int_{\Gamma_{1}}^{\text {on } \Gamma_{0}^{+}} \mathbf{G}_{\mathrm{uu}} \mathbf{f}_{1}+\int_{\Gamma_{2}} \mathbf{G}_{\mathrm{ut}}\left(-\mathbf{u}_{2}\right)+\int_{\Gamma_{0}} \mathbf{G}_{\mathrm{uu}} \mathbf{f}_{0}+\oint_{\Gamma_{0}} \mathbf{G}_{\mathrm{ut}}\left(-\mathbf{u}_{0}\right)-\frac{1}{2} \mathbf{u}_{0} \\
& \quad+\int_{\Gamma_{1}} \mathbf{G}_{\mathrm{ut}}\left(-\overline{\mathbf{u}}_{1}\right)+\int_{\Gamma_{2}} \mathbf{G}_{\mathrm{uu}} \overline{\mathbf{f}}_{2}+\int_{\Omega} \mathbf{G}_{\mathrm{uu}} \overline{\mathbf{b}}+\int_{\Omega} \mathbf{G}_{\mathrm{u} \sigma} \overline{\boldsymbol{\vartheta}}=0
\end{aligned}
$$

and on $\Gamma_{0}$

$$
\begin{aligned}
\mathbf{t}_{0} & =\int_{\Gamma_{1}} \mathbf{G}_{\mathrm{tu}} \mathbf{f}_{1}+\int_{\Gamma_{2}} \mathbf{G}_{\mathrm{tt}}\left(-\mathbf{u}_{2}\right)+\oint_{\Gamma_{0}} \mathbf{G}_{\mathrm{tu}} \mathbf{f}_{0}+\frac{1}{2} \mathbf{f}_{0}+\int_{\Gamma_{0}} \mathbf{G}_{\mathrm{tt}}\left(-\mathbf{u}_{0}\right) \\
& +\int_{\Gamma_{1}} \mathbf{G}_{\mathrm{tt}}\left(-\overline{\mathbf{u}}_{1}\right)+\int_{\Gamma_{2}} \mathbf{G}_{\mathrm{tu}} \overline{\mathbf{f}}_{2}+\int_{\Omega} \mathbf{G}_{\mathrm{tu}} \overline{\mathbf{b}}+\int_{\Omega} \mathbf{G}_{\mathrm{t} \sigma} \overline{\boldsymbol{\vartheta}}
\end{aligned}
$$

on $\Gamma_{2}$ 
Let us introduce the boundary discretization through boundary elements by carrying out the following variable modelling:

$\mathbf{f}_{1}=\Psi_{\mathrm{f}} \mathbf{F}_{1}$

$\mathbf{u}_{2}=\Psi_{\mathrm{u}} \mathbf{U}_{2}$

$\mathbf{f}_{0}=\Psi_{\mathrm{f}} \mathbf{F}_{0}$

$\mathbf{u}_{0}=\Psi_{\mathrm{u}} \mathbf{U}_{0}$,

where the capital letters define the nodal values of the reactions $\left(\mathbf{F}_{1}\right.$ on $\Gamma_{1}$ and $\mathbf{F}_{0}$ on $\left.\Gamma_{0}\right)$ and of the displacements $\left(\mathbf{U}_{2}\right.$ on $\Gamma_{2}$ and $\mathbf{U}_{0}$ on $\left.\Gamma_{0}\right)$.

Let us now perform the weighting of all the coefficients of the Eqs. (5a)-(5d). For this purpose, the same shape functions as those modelling the response have been employed, but in an energetically dual way on the base of the Galerkin approach: the coefficients defining displacements are weighted through the shape functions modelling the forces, and vice-versa the coefficients defining tractions are weighted through the shape functions modelling the displacements. In this way it is possible to obtain the following block system

$$
\left|\begin{array}{c}
\mathbf{W}_{1}^{+}=\mathbf{0} \\
\mathbf{P}_{2}^{+}=\mathbf{0} \\
\mathbf{W}_{0}^{+}=\mathbf{0} \\
\hdashline \mathbf{P}_{0}
\end{array}\right|=\left|\begin{array}{ccc:c}
\mathbf{A}_{\mathrm{u} 1, \mathrm{u} 1} & \mathbf{A}_{\mathrm{u} 1, \mathrm{f} 2} & \mathbf{A}_{\mathrm{u} 1, \mathrm{u} 0} & \mathbf{A}_{\mathrm{u} 1, \mathrm{f} 0} \\
\mathbf{A}_{\mathrm{f} 2, \mathrm{u} 1} & \mathbf{A}_{\mathrm{f} 2, \mathrm{f} 2} & \mathbf{A}_{\mathrm{f} 2, \mathrm{u} 0} & \mathbf{A}_{\mathrm{f} 2, \mathrm{f} 0} \\
\mathbf{A}_{\mathrm{u} 0, \mathrm{u} 1} & \mathbf{A}_{\mathrm{u} 0, \mathrm{f} 2} & \mathbf{A}_{\mathrm{u} 0, \mathrm{u} 0} & \overline{\mathbf{A}}_{\mathrm{u} 0, \mathrm{f} 0} \\
\hdashline \mathbf{A}_{\mathrm{f} 0, \mathrm{u} 1} & \mathbf{A}_{\mathrm{f} 0, \mathrm{f} 2} & \overline{\mathbf{A}}_{\mathrm{f} 0, \mathrm{u} 0} & \mathbf{A}_{\mathrm{f} 0, \mathrm{f} 0}
\end{array}\right|\left|\begin{array}{c}
\mathbf{F}_{1} \\
-\mathbf{U}_{2} \\
\mathbf{F}_{0}
\end{array}\right|+\mid \begin{gathered}
\hat{\mathbf{W}}_{1}^{+} \\
\hat{\mathbf{P}}_{2}^{+} \\
\hat{\mathbf{W}}_{0}^{+} \\
\hdashline \hat{\mathbf{L}}_{0}
\end{gathered}
$$

In the latter equation the conditions $\mathbf{W}_{1}^{+}=\int_{\Gamma_{1}^{+}} \tilde{\psi}_{\mathrm{f}} \mathbf{u}_{1}^{+}=0$ and $\mathbf{P}_{2}^{+}=\int_{\Gamma_{2}^{+}} \tilde{\psi}_{\mathrm{u}} \mathbf{t}_{2}^{+}=0$ have to be considered as the classical Diriclet and Neumann conditions of the continuum $\left(\mathbf{u}_{1}=\overline{\mathbf{u}}_{1}\right.$ on $\Gamma_{1}$ and $\mathbf{t}_{2}=\overline{\mathbf{f}}_{2}$ on $\Gamma_{2}$, or, which is the same thing, $\mathbf{u}_{1}^{+}=0$ on $\Gamma_{1}^{+}$and $\mathbf{t}_{2}^{+}=\mathbf{0}$ on $\Gamma_{2}^{+}$), written on the boundary elements $\Gamma_{1}^{+}$and $\Gamma_{2}^{+}$of the complementary domain, respectively, in terms of generalized (or weighted) quantities. The other two expressions regard quantities of the interface zone: specifically, the condition $\mathbf{W}_{0}^{+}=\int_{\Gamma_{0}^{+}} \tilde{\psi}_{\mathrm{f}} \mathbf{u}_{0}=0$ represents the generalized displacement, null on the boundary $\Gamma_{0}^{+}$, whereas the term $\mathbf{P}_{0}=\int_{\Gamma_{0}} \tilde{\psi}_{\mathrm{u}} \mathbf{t}_{0}$ collects the values of the generalized tractions defined on the boundary elements of $\Gamma_{0}$.

In Eq. (7) the following positions have been set for the matrix coefficients

$$
\begin{aligned}
\widehat{\mathbf{P}}_{2}^{+}= & \int_{\Gamma_{2}^{+}} \tilde{\psi}_{\mathrm{u}} \int_{\Gamma_{1}} \mathbf{G}_{\mathrm{tt}} \psi_{\mathrm{u}}\left(-\overline{\mathbf{U}}_{1}\right)+\int_{\Gamma_{2}^{+}} \tilde{\psi}_{\mathrm{u}} \oint_{\Gamma_{2}} \mathbf{G}_{\mathrm{tu}} \psi_{\mathrm{f}} \overline{\mathbf{F}}_{2} \\
& -\frac{1}{2} \int_{\Gamma_{2}^{+}} \tilde{\psi}_{\mathrm{u}} \psi_{\mathrm{f}} \overline{\mathbf{F}}_{2}+\int_{\Gamma_{2}^{+}} \tilde{\psi}_{\mathrm{u}} \int_{\Omega} \mathbf{G}_{\mathrm{tu}} \overline{\mathbf{b}}+\int_{\Gamma_{2}^{+}} \tilde{\psi}_{\mathrm{u}} \int_{\Omega} \mathbf{G}_{\mathrm{t} \sigma} \overline{\boldsymbol{\vartheta}} \\
\widehat{\mathbf{W}}_{0}^{+}= & \int_{\Gamma_{0}^{+}} \tilde{\psi}_{\mathrm{f}} \int_{\Gamma_{1}} \mathbf{G}_{\mathrm{ut}} \psi_{\mathrm{u}}\left(-\overline{\mathbf{U}}_{1}\right)+\int_{\Gamma_{0}^{+}} \tilde{\psi}_{\mathrm{f}} \int_{\Gamma_{2}} \mathbf{G}_{\mathrm{uu}} \psi_{\mathrm{f}} \overline{\mathbf{F}}_{2} \\
& +\int_{\Gamma_{0}^{+}} \tilde{\psi}_{\mathrm{f}} \int_{\Omega} \mathbf{G}_{\mathrm{uu}} \overline{\mathbf{b}}+\int_{\Gamma_{0}^{+}} \tilde{\psi}_{\mathrm{f}} \int_{\Omega} \mathbf{G}_{\mathrm{u} \sigma} \overline{\boldsymbol{\vartheta}} \\
\widehat{\mathbf{L}}_{0}= & \int_{\Gamma_{0}} \tilde{\psi}_{\mathrm{u}} \int_{\Gamma_{1}} \mathbf{G}_{\mathrm{tt}} \psi_{\mathrm{u}}\left(-\overline{\mathbf{U}}_{1}\right)+\int_{\Gamma_{0}} \tilde{\psi}_{\mathrm{u}} \int_{\Gamma_{2}} \mathbf{G}_{\mathrm{tu}} \psi_{\mathrm{f}} \overline{\mathbf{F}}_{2} \\
& +\int_{\Gamma_{0}} \tilde{\psi}_{\mathrm{u}} \int_{\Omega} \mathbf{G}_{\mathrm{tu}} \overline{\mathbf{b}}+\int_{\Gamma_{0}} \tilde{\psi}_{\mathrm{u}} \int_{\Omega} \mathbf{G}_{\mathrm{t} \sigma} \overline{\boldsymbol{\vartheta}} .
\end{aligned}
$$

In Eq. (8j), seen previously, the terms $\overline{\mathbf{A}}_{\mathrm{u}_{0}, \mathrm{f}_{0}}$ and $\tilde{\overline{\mathbf{A}}}_{\mathrm{f}_{0}, \mathrm{u}_{0}}$ include the weighting of the CPV integrals and of the corresponding free terms.

As can easily be observed, in positions $(8,9)$ the coefficients are made up of double integrals. Some of the integrals constituting the matrix $\mathbf{A}$ in Eq. (7) show singular or hypersingular kernels: this happens when the boundary cause distribution acts on the boundary elements where the weighted effect is valued.

Panzeca et al. (2001) have removed the computational difficulties mentioned by determining all the coefficients of the matrix $\mathbf{A}$ in closed form, both those containing singular or hypersingular kernels and those computed as Riemann integrals, thus producing high performance. The load vector coefficients are obtained in closed form too. A particular strategy made it possible to set up a software, called Karnak.sGbem (Panzeca et al., 2002a), able to give solutions to plane structural system having any geometry and subdivisible into bem-es having different material characteristics and different thicknesses. These systems may be subjected to inplane external actions, like boundary and volume forces or imposed displacements of the constraints and inelastic actions.

On the basis of these considerations, Eq. (7) may be expressed in extended form with obvious meaning of the symbols in the following way:

$$
\begin{aligned}
& \mathbf{A}_{\mathrm{u}_{1}, \mathrm{u}_{1}}=\int_{\Gamma_{1}^{+}} \tilde{\psi}_{\mathrm{f}} \int_{\Gamma_{1}} \mathbf{G}_{\mathrm{uu}} \psi_{\mathrm{f}}=\tilde{\mathbf{A}}_{u_{1}, u_{1}}, \mathbf{A}_{u_{1}, f_{2}}=\int_{\Gamma_{1}^{+}} \tilde{\psi}_{f} \int_{\Gamma_{2}} \mathbf{G}_{\mathrm{ut}} \psi_{\mathrm{u}}=\tilde{\mathbf{A}}_{f_{2}, u_{1}}, \mathbf{A}_{f_{2}, f_{2}}=\int_{\Gamma_{2}^{+}} \tilde{\psi}_{\mathrm{u}} \int_{\Gamma_{2}} \mathbf{G}_{\mathrm{tt}} \psi_{\mathrm{u}}=\tilde{\mathbf{A}}_{f_{2}, f_{2}}, \\
& \mathbf{A}_{u_{1}, u_{0}}=\int_{\Gamma_{1}^{+}} \tilde{\psi}_{\mathrm{f}} \int_{\Gamma_{0}} \mathbf{G}_{\mathrm{uu}} \psi_{\mathrm{f}}=\tilde{\mathbf{A}}_{u_{0}, u_{1}}, \quad \mathbf{A}_{f_{2}, u_{0}}=\int_{\Gamma_{2}^{+}} \tilde{\psi}_{\mathrm{u}} \int_{\Gamma_{0}} \mathbf{G}_{\mathrm{tu}} \psi_{\mathrm{f}}=\tilde{\mathbf{A}}_{u_{0}, f_{2}}, \mathbf{A}_{u_{0}, u_{0}}=\int \tilde{\psi}_{\mathrm{f}} \int_{\Gamma_{0}} \mathbf{G}_{\mathrm{uu}} \psi_{\mathrm{f}}=\tilde{\mathbf{A}}_{u_{0}, u_{0}}, \\
& \mathbf{A}_{\mathrm{u}_{1}, \mathrm{f}_{0}}=\int_{\Gamma_{1}^{+}} \tilde{\psi}_{f} \int_{\Gamma_{2}} \mathbf{G}_{\mathrm{tu}} \psi_{\mathrm{f}}=\tilde{\mathbf{A}}_{f_{0}, u_{1}}, \mathbf{A}_{f_{2}, f_{0}}=\int_{\Gamma_{2}^{+}} \tilde{\psi}_{\mathrm{u}} \int_{\Gamma_{0}} \mathbf{G}_{\mathrm{tt}} \psi_{\mathrm{u}}=\tilde{\mathbf{A}}_{f_{0}, f_{2}}, \mathbf{A}_{f_{0}, f_{0}}=\iint_{\Gamma_{0}} \tilde{\psi}_{\mathrm{u}} \int_{\Gamma_{0}} \mathbf{G}_{\mathrm{tt}} \psi_{\mathrm{u}}=\tilde{\mathbf{A}}_{f_{0}, f_{0}}, \\
& \overline{\mathbf{A}}_{u_{0}, f_{0}}=\int_{\Gamma_{0}^{+}} \tilde{\psi}_{\mathrm{f}} \oint_{\Gamma_{0}} \mathbf{G}_{\mathrm{ut}} \psi_{\mathrm{u}}+\frac{1}{2} \int_{\Gamma_{0}^{+}} \tilde{\psi}_{\mathrm{f}} \psi_{\mathrm{u}}=\tilde{\overline{\mathbf{A}}}_{f_{0}, u_{0}},
\end{aligned}
$$

and for the load vector

$$
\begin{aligned}
\widehat{\mathbf{W}}_{1}^{+} & =\int_{\Gamma_{1}^{+}} \tilde{\psi}_{\mathrm{f}} \oint_{\Gamma_{1}} \mathbf{G}_{\mathrm{ut}} \psi_{\mathrm{u}}\left(-\overline{\mathbf{U}}_{1}\right)+\frac{1}{2} \int_{\Gamma_{1}^{+}} \tilde{\psi}_{\mathrm{f}} \psi_{\mathrm{u}}\left(-\overline{\mathbf{U}}_{1}\right) \\
& +\int_{\Gamma_{1}^{+}} \tilde{\psi}_{\mathrm{f}} \int_{\Gamma_{2}} \mathbf{G}_{\mathrm{uu}} \psi_{\mathrm{f}} \overline{\mathbf{F}}_{2}+\int_{\Gamma_{1}^{+}} \tilde{\psi}_{\mathrm{f}} \int_{\Omega} \mathbf{G}_{\mathrm{uu}} \overline{\mathbf{b}}+\int_{\Gamma_{1}^{+}} \tilde{\psi}_{\mathrm{f}} \int_{\Omega} \mathbf{G}_{\mathrm{u} \sigma} \overline{\boldsymbol{\vartheta}}
\end{aligned}
$$

$$
\begin{aligned}
& \mathbf{0}=\mathbf{A} \mathbf{X}+\mathbf{A}_{0}\left(-\mathbf{U}_{0}\right)+\widehat{\mathbf{L}} \\
& \mathbf{P}_{0}=\tilde{\mathbf{A}}_{0} \mathbf{X}+\mathbf{A}_{00}\left(-\mathbf{U}_{0}\right)+\widehat{\mathbf{L}}_{0}
\end{aligned}
$$

where the vector $\mathbf{X}$ collects the boundary sub-vectors $\mathbf{F}_{1},\left(-\mathbf{U}_{2}\right)$ and $\mathbf{F}_{0}$, whereas $\left(-\mathbf{U}_{0}\right)$ collects the nodal displacements in the interface zone, changed in sign. The vector $\mathbf{P}_{0}$ represents the generalized (or weighted) traction vector defined in the boundary elements of the same interface zone, obtained as a weighted response to all the 


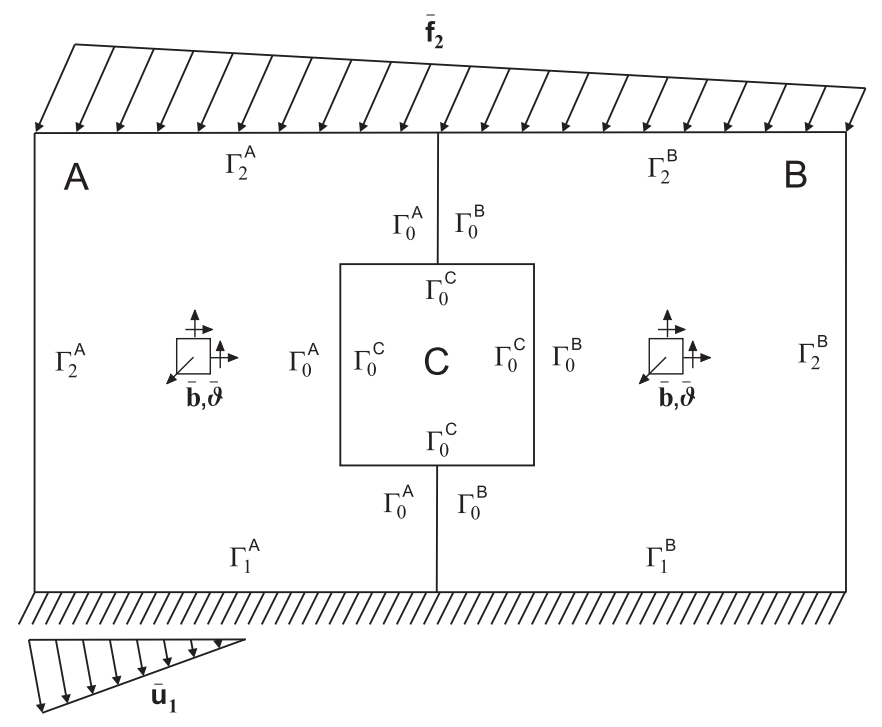

Fig. 1. System subdivided into bem-es A, B, and C.

known and unknown actions, regarding boundary and volume quantities.

By performing a variable condensation through the replacement of the $\mathbf{X}$ vector extracted from Eq. (10a) in Eq. (10b), we obtain the relevant equation:

$\mathbf{P}_{0}=\mathbf{D}_{00} \mathbf{U}_{0}+\widehat{\mathbf{P}}_{0}$

where the following positions have been made:

$\mathbf{D}_{00}=\tilde{\mathbf{A}}_{0} \mathbf{A}^{-1} \mathbf{A}_{0}-\mathbf{A}_{00}$,

$\widehat{\mathbf{P}}_{0}=\widehat{\mathbf{L}}_{0}-\tilde{\mathbf{A}}_{0} \mathbf{A}^{-1} \widehat{\mathbf{L}}$.

Eq. (11), called characteristic equation of the bem-e, relates the generalized (or weighted) tractions $\mathbf{P}_{0}$ defined on the elements of the boundary in the interface zone $\Gamma_{0}$ to the displacements $\mathbf{U}_{0}$ of the nodes of the same boundary and to the load term $\widehat{\mathbf{P}}_{0}$, the latter being the generalized traction vector, as the effect evaluated on the same interface boundary elements, caused by the external actions present in the bem-e being examined. Furthermore, $\mathbf{D}_{00}$ is the related stiffness matrix.

As can be shown, Eq. (11), valid for each bem-e, is formally identical to that written in the sphere of the FEM (Cook, 1989).

The previous considerations show the high performance that this approach is able to give. Indeed, among the main characteristics we can mention the following:
- computation in closed form of all the coefficients of the stiffness matrix and of the load vector in Eq. (11); and

- very simple modification of the algebraic operators dimensions involved, which is useful in nonlinear analysis.

\subsection{Solving equation system}

In order to obtain the elastic response of a structure subjected to known external actions, the same two-dimensional continuum body as in Fig. 1 was considered. As a consequence of the physical $(E, \nu)$ and geometrical $(s)$ characteristics, it is subdivided into three subdomains specified by the capital Latin letters A, B, C.

The bem-e interfaces are called by the lower-case Latin letters a, b and $\mathrm{c}$ between the solids A and B, A and C, B and C, respectively. The two nodes indicated by $\mathrm{H}$ belong to all the bem-es as shown in Fig. 3.

Each of the bem-es A, B and C is characterized by a relationship between the generalized tractions and the nodal displacements, both quantities defined on $\Gamma_{0}$ as shown in Eq. (11). For example, for the bem-e A we can write in detail:

$$
\left|\begin{array}{l}
\mathbf{P}_{0 a}^{A} \\
\mathbf{P}_{0 b}^{A} \\
\mathbf{P}_{0 H}^{A}
\end{array}\right|=\left|\begin{array}{lll}
\mathbf{D}_{0 a, 0 a}^{A} & \mathbf{D}_{0 a, 0 b}^{A} & \mathbf{D}_{0 a, 0 H}^{A} \\
\mathbf{D}_{0 b, 0 a}^{A} & \mathbf{D}_{0 b, 0 b}^{A} & \mathbf{D}_{0 b, 0 H}^{A} \\
\mathbf{D}_{0 H, 0 a}^{A} & \mathbf{D}_{0 H, 0 b}^{A} & \mathbf{D}_{0 H, 0 H}^{A}
\end{array}\right|\left|\begin{array}{c}
\mathbf{U}_{0 a}^{A} \\
\mathbf{U}_{0 b}^{A} \\
\mathbf{U}_{0 H}^{A}
\end{array}\right|+\left|\begin{array}{c}
\widehat{\mathbf{P}}_{0 a}^{A} \\
\widehat{\mathbf{P}}_{0 b}^{A} \\
\widehat{\mathbf{P}}_{0 H}^{A}
\end{array}\right|
$$

Let us introduce the characteristic equation for each bem-e:

$\mathbf{P}_{0}^{A}=\mathbf{D}_{00}^{A} \mathbf{U}_{0}^{A}+\widehat{\mathbf{P}}_{0}^{A}$ for body A

$\mathbf{P}_{0}^{\mathrm{B}}=\mathbf{D}_{00}^{B} \mathbf{U}_{0}^{B}+\widehat{\mathbf{P}}_{0}^{B} \quad$ for body B

$\mathbf{P}_{0}^{C}=\mathbf{D}_{00}^{C} \mathbf{U}_{0}^{C}+\widehat{\mathbf{P}}_{0}^{C}$ for body $\mathrm{C}$

Now imposing the regularity conditions for the displacements of the interface nodes

$\mathbf{U}_{0 k}=\mathbf{U}_{0 k}^{i}=\mathbf{U}_{0 k}^{j}$

and for the generalized tractions of the interface boundary elements

$\mathbf{P}_{0 k}^{i}=-\mathbf{P}_{0 k}^{j}$

where $i, j=\mathrm{A}, \mathrm{B}, \mathrm{C}, i \neq j$ and $k=\mathrm{a}, \mathrm{b}, \mathrm{c}, \mathrm{H}$ we obtain the elastic solution in terms of the nodal displacements $\mathbf{U}_{0}$ through the following solving equation system:

$\mathbf{K}_{00} \mathbf{U}_{0}+\widehat{\mathbf{f}}_{0}=\mathbf{0}$

where the following positions have been set

$$
\begin{gathered}
\mathbf{K}_{00}=\begin{array}{ccc:c}
\left(\mathbf{D}_{0 a, 0 a}^{A}+\mathbf{D}_{0 a, 0 a}^{B}\right) & \mathbf{D}_{0 a, 0 b}^{A} & \mathbf{D}_{0 a, 0 c}^{B} & \left(\mathbf{D}_{0 a, 0 H}^{A}+\mathbf{D}_{0 a, 0 H}^{B}\right) \\
\mathbf{D}_{0 b, 0 a}^{A} & \left(\mathbf{D}_{0 b, 0 b}^{A}+\mathbf{D}_{0 b, 0 b}^{C}\right) & \mathbf{D}_{0 b, 0 c}^{C} & \left(\mathbf{D}_{0 b, 0 H}^{A}+\mathbf{D}_{0 b, 0 H}^{C}\right) \\
\mathbf{D}_{0 c, 0 a}^{B} & \mathbf{D}_{0 c, 0 b}^{C} & \left(\mathbf{D}_{0 c, 0 c}^{B}+\mathbf{D}_{0 c, 0 c}^{C}\right) & \left(\mathbf{D}_{0 c, 0 H}^{B}+\mathbf{D}_{0 c, 0 H}^{C}\right) \\
\hdashline\left(\mathbf{D}_{0 H, 0 a}^{A}+\mathbf{D}_{0 H, 0 a}^{B}\right) & \left(\mathbf{D}_{0 H, 0 b}^{A}+\mathbf{D}_{0 H, 0 b}^{C}\right) & \left(\mathbf{D}_{0 H, 0 c}^{B}+\mathbf{D}_{0 H, 0 c}^{C}\right) & \left(\mathbf{D}_{0 H, 0 H}^{A}+\mathbf{D}_{0 H, 0 H}^{B}+\mathbf{D}_{0 H, 0 H}^{C}\right)
\end{array} \mid \\
\hat{\mathbf{f}}_{0}^{T}=\mid\left(\hat{\mathbf{P}}_{0 a}^{A}+\hat{\mathbf{P}}_{0 a}^{B}\right)^{T} \\
\left(\hat{\mathbf{P}}_{0 b}^{A}+\hat{\mathbf{P}}_{0 b}^{C}\right)^{T} \\
\left(\hat{\mathbf{P}}_{0 c}^{B}+\hat{\mathbf{P}}_{0 c}^{C}\right)^{T}\left|\left(\hat{\mathbf{P}}_{0 H}^{A}+\hat{\mathbf{P}}_{0 H}^{B}+\hat{\mathbf{P}}_{0 H}^{C}\right)^{T}\right| \\
\mathbf{U}_{0}^{T}=\mid \mathbf{U}_{0 a}^{T} \mathbf{U}_{0 b}^{T} \\
\mathbf{U}_{0 c}^{T}\left|\mathbf{U}_{0 H}^{T}\right|
\end{gathered}
$$




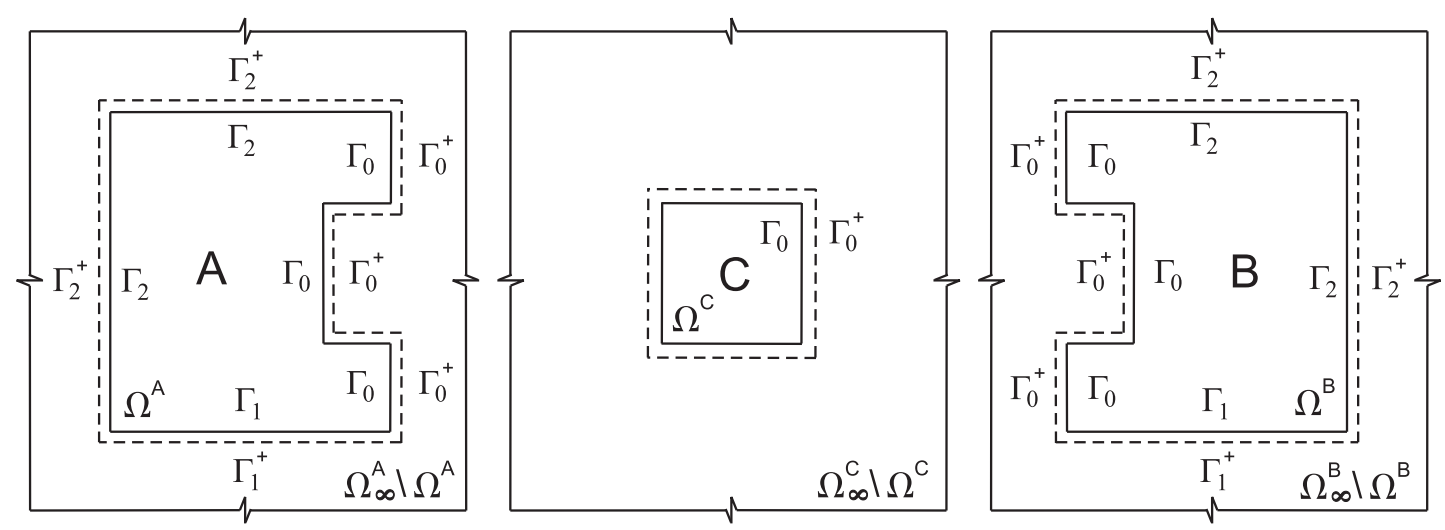

Fig. 2. Bem-es A, B, and C, each embedded in $\Omega_{\infty}$.

The remaining boundary quantities of the substructures $\mathrm{A}, \mathrm{B}$ and $C$, regarding the nodal reactive forces $F_{1}$ of the constrained boundary $\Gamma_{1}$, the displacements $\mathbf{U}_{2}$ of the free nodes of $\Gamma_{2}$ and the nodal interface forces $\mathbf{F}_{0}$ between the bem-es, all variable quantities collected in the vectors $\mathbf{X}^{\mathrm{A}}, \mathbf{X}^{\mathrm{B}}$ and $\mathbf{X}^{\mathrm{C}}$, are obtainable by using equations like Eq. (10a), written for each bem-e.

\section{Displacement approach with external unknowns}

In this section a new displacement approach is presented which reduces the unknowns in the analysis phase. It is possible, if one wishes, to utilize as variables not the nodal displacements $\mathbf{U}_{0}$ of all the interface boundaries $\Gamma_{0}$, but the nodal displacements $\mathbf{U}_{2}$ of the free boundary $\Gamma_{2}$ of the assembled system.

Let us define an appropriate relation for each bem-e, deduced by Eq. (7), i.e.:

$$
\begin{aligned}
& \mathbf{P}_{20}=\mathbf{D}_{20,20} \mathbf{U}_{20}+\underbrace{\widehat{\mathbf{P}}_{\Gamma}(\overline{\mathbf{f}}, \overline{\mathbf{u}})+\widehat{\mathbf{P}}_{\Omega}(\overline{\mathbf{b}}, \overline{\boldsymbol{\vartheta}})}_{\widehat{P}_{20}} \\
& \text { where } \mathbf{P}_{20}=\left|\begin{array}{c}
\mathbf{P}_{2}^{+}=\mathbf{0} \\
\mathbf{P}_{0}
\end{array}\right|, \quad \mathbf{U}_{20}=\left|\begin{array}{l}
\mathbf{U}_{2} \\
\mathbf{U}_{0}
\end{array}\right|
\end{aligned}
$$

This relation connects the generalized tractions $\mathbf{P}_{2}^{+}=\mathbf{0}$ and $\mathbf{P}_{0}$ introduced to define Eq. (7) (the first vector assumed to be null according to the generalized Neumann condition) along the boundaries $\Gamma_{2}^{+}$and $\Gamma_{0}$ to the nodal displacements $\mathbf{U}_{2}$ and $\mathbf{U}_{0}$ of the boundaries $\Gamma_{2}$ and $\Gamma_{0}$, through the stiffness matrix $\mathbf{D}_{20,20}$ and to the known generalized traction vectors $\widehat{\mathbf{P}}_{\Gamma}$ and $\widehat{\mathbf{P}}_{\Omega}$.

The use of appropriate regularity conditions at the interface boundaries leads to the following solving equations system, here defined as a complete solution:

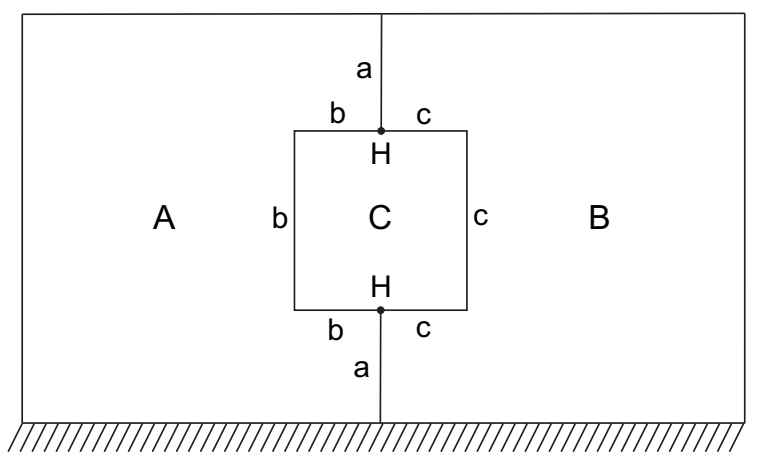

Fig. 3. Characterization of the interface boundaries.
$\mathbf{K}_{20,20} \mathbf{U}_{20}+\widehat{\mathbf{f}}_{20}=\mathbf{0}$

where the unknown vector $\mathbf{U}_{20}$ collects the displacements $\mathbf{U}_{2}$ and $\mathbf{U}_{0}$ of all the nodes belonging to the boundaries $\Gamma_{2}$ and $\Gamma_{0}$, in full analogy with the FEM.

A further variable condensation process in the previously assembled system leads to the following solving equation system in the displacement approach where only the external unknowns are present:

$\mathbf{H}_{22} \mathbf{U}_{2}+\widehat{\mathbf{t}}_{2}=\mathbf{0}$

In it the unknowns are the nodal displacements $\mathbf{U}_{2}$ of all the free boundaries of the assembled system, obtained through the symmetric stiffness matrix $\mathbf{H}_{22}$ and the load vector $\widehat{\mathbf{t}}_{2}$.

In the following sub-sections both the rise of the elasticity relation of each bem-e and the solving equations system are shown.

\subsection{The elasticity relation of each bem-e}

Let us perform a different arrangement of the block system of Eq. (7), as follows:

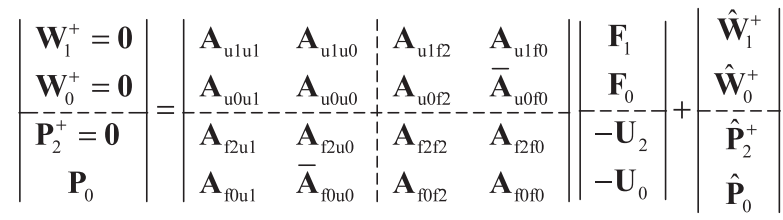

The meaning of all the block coefficients remains unchanged.

The previous equation may be expressed in compact form as follows with obvious meaning of symbols:

$$
\begin{aligned}
& \mathbf{0}=\mathbf{A}_{\mathrm{uu}} \mathbf{F}_{10}+\mathbf{A}_{\mathrm{uf}}\left(-\mathbf{U}_{20}\right)+\widehat{\mathbf{L}}_{\mathrm{u}} \\
& \mathbf{P}_{20}=\tilde{\mathbf{A}}_{\mathrm{fu}} \mathbf{F}_{10}+\mathbf{A}_{\mathrm{ff}}\left(-\mathbf{U}_{20}\right)+\widehat{\mathbf{L}}_{\mathrm{f}}
\end{aligned}
$$

The vector $\mathbf{P}_{20}$ collects the generalized tractions sub-vectors evaluated on the free boundary elements of $\Gamma_{2}^{+}$and the interface ones of $\Gamma_{0}$.

Let us perform a first variable condensation by substituting the vector $\mathbf{F}_{10}$, obtained by Eq. (23a), in Eq. (23b). One obtains the following block system written for each bem-e:

$$
\left|\begin{array}{c}
\mathbf{0} \\
\hdashline \mathbf{P}_{0}
\end{array}\right|=\left|\begin{array}{c:c}
\mathbf{D}_{22} & \mathbf{D}_{20} \\
\hdashline \mathbf{D}_{02} & \mathbf{D}_{00}
\end{array}\right|\left|\mathbf{U}_{2}\right|\left|\begin{array}{c}
\hat{\mathbf{U}}_{0} \\
\hdashline \mid \hat{\mathbf{P}}_{0}
\end{array}\right|
$$


or in compact form

$\mathbf{P}_{20}=\mathbf{D}_{20,20} \mathbf{U}_{20}+\widehat{\mathbf{P}}_{20}$

where the following positions have been made:

$\mathbf{D}_{20,20}=\tilde{\mathbf{A}}_{\mathrm{fu}} \mathbf{A}_{\mathrm{uu}}^{-1} \mathbf{A}_{\mathrm{uf}}-\mathbf{A}_{\mathrm{ff}}$,

$\widehat{\mathbf{P}}_{20}=\widehat{\mathbf{L}}_{\mathrm{f}}-\tilde{\mathbf{A}}_{\mathrm{fu}} \mathbf{A}_{\mathrm{uu}}^{-1} \widehat{\mathbf{L}}_{\mathrm{u}}$

Eq. (25) is the characteristic equation of each bem-e. This connects the generalized tractions $\mathbf{P}_{2}^{+}=0$ and $\mathbf{P}_{0}$ evaluated on the boundary elements of $\Gamma_{2}^{+}$and $\Gamma_{0}$ to the nodal displacements of the boundary $\Gamma_{2}$ and $\Gamma_{0}$ through the symmetric stiffness matrix $\mathbf{D}_{20,20}$ and the load vector $\widehat{\mathbf{P}}_{20}$. and of generalized equilibrium at the same interface boundary elements, analogously to what was shown in Section 3.2, i.e.

$\mathbf{P}_{0 k}^{i}=-\mathbf{P}_{0 k}^{j}$

where $i, j=\mathrm{A}, \mathrm{B}, \mathrm{C}, i \neq j$ and $k=\mathrm{a}, \mathrm{b}, \mathrm{c}, \mathrm{H}$.

Let us obtain a solving system where the only unknowns are the free $\mathbf{U}_{2}$ and interface $\mathbf{U}_{0}$ nodal displacements:

$\mathbf{K}_{20,20} \mathbf{U}_{20}+\widehat{\mathbf{f}}_{20}=\mathbf{0}$

where

$$
\begin{aligned}
& \mathbf{K}_{20,20}=\left|\begin{array}{cc:ccc:c}
\mathbf{D}_{2,2}^{A} & \mathbf{0} & \mathbf{D}_{2,0 a}^{A} & \mathbf{D}_{2,0 b}^{A} & \mathbf{0} & \mathbf{D}_{2,0 H}^{A} \\
\mathbf{0} & \mathbf{D}_{2,2}^{B} & \mathbf{D}_{2,0 a}^{B} & \mathbf{0} & \mathbf{D}_{2,0 c}^{B} & \mathbf{D}_{2,0 H}^{B} \\
\hdashline \mathbf{D}_{0 a, 2}^{A} & \mathbf{D}_{0 a, 2}^{B} & \left(\mathbf{D}_{0 a, 0 a}^{A}+\mathbf{D}_{0 a, 0 a}^{B}\right) & \mathbf{D}_{0 a, 0 b}^{A} & \mathbf{D}_{0 a, 0 c}^{B} & \left(\mathbf{D}_{0 a, 0 H}^{A}+\mathbf{D}_{0 a, 0 H}^{B}\right) \\
\mathbf{D}_{0 b, 2}^{A} & \mathbf{0} & \mathbf{D}_{0 b, 0 a}^{A} & \left(\mathbf{D}_{0 b, 0 b}^{A}+\mathbf{D}_{0 b, 0 b}^{C}\right) & \mathbf{D}_{0 b, 0 c}^{C} & \left(\mathbf{D}_{0 b, 0 H}^{A}+\mathbf{D}_{0 b, 0 H}^{C}\right) \\
\mathbf{0} & \mathbf{D}_{0 c, 2}^{B} & \mathbf{D}_{0 c, 0 a}^{B} & \mathbf{D}_{0 c, 0 b}^{C} & \left(\mathbf{D}_{0 c, 0 c}^{B}+\mathbf{D}_{0 c, 0 c}^{C}\right) & \left(\mathbf{D}_{0 c, 0 H}^{B}+\mathbf{D}_{0 c, 0 H}^{C}\right) \\
\hdashline \mathbf{D}_{0 H, 2}^{A} & \mathbf{D}_{0 H, 2}^{B} & \left(\mathbf{D}_{0 H, 0 a}^{A}+\mathbf{D}_{0 H, 0 a}^{B}\right) & \left(\mathbf{D}_{0 H, 0 b}^{A}+\mathbf{D}_{0 H, 0 b}^{C}\right) & \left(\mathbf{D}_{0 H, 0 c}^{B}+\mathbf{D}_{0 H, 0 c}^{C}\right) & \left(\mathbf{D}_{0 H, 0 H}^{A}+\mathbf{D}_{0 H, 0 H}^{B}+\mathbf{D}_{0 H, 0 H}^{C}\right)
\end{array}\right|
\end{aligned}
$$

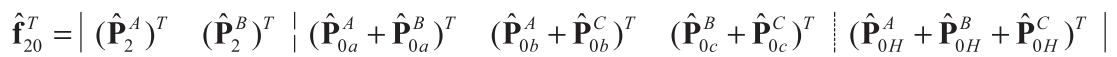

$$
\begin{aligned}
& \mathbf{U}_{20}^{T}=\left|\begin{array}{ll:lll:l}
\left(\mathbf{U}_{2}^{A}\right)^{T} & \left(\mathbf{U}_{2}^{B}\right)^{T} & \mathbf{U}_{0 a}^{T} & \mathbf{U}_{0 b}^{T} & \mathbf{U}_{0 c}^{T} & \mathbf{U}_{0 H}^{T}
\end{array}\right|
\end{aligned}
$$

\subsection{Solving equation system}

In order to obtain the elastic response of the structure, subdivided into bem-es and subjected to external actions, the bidimensional solid in Fig. 3 is considered.

Each bem-e A, B and C is characterized by a characteristic relation which connects generalized tractions to nodal displacements (Eq. (25)). For example, for the bem-e A we can write:

$$
\left|\begin{array}{c}
\left(\mathbf{P}_{2}^{+}\right)^{A}=\mathbf{0} \\
\hdashline \mathbf{P}_{0 a}^{A} \\
\mathbf{P}_{0 b}^{A} \\
\mathbf{P}_{0 H}^{A}
\end{array}\right|=\left|\begin{array}{c:ccc}
\mathbf{D}_{2,2}^{A} & \mathbf{D}_{2,0 a}^{A} & \mathbf{D}_{2,0 b}^{A} & \mathbf{D}_{2,0 H}^{A} \\
\hdashline \mathbf{D}_{0 a, 2}^{A} & \mathbf{D}_{0 a, 0 a}^{A} & \mathbf{D}_{0 a, 0 b}^{A} & \mathbf{D}_{0 a, 0 H}^{A} \\
\mathbf{D}_{0 b, 2}^{A} & \mathbf{D}_{0 b, 0 a}^{A} & \mathbf{D}_{0 b, 0 b}^{A} & \mathbf{D}_{0 b, 0 H}^{A} \\
\mathbf{D}_{0 H, 2}^{A} & \mathbf{D}_{0 H, 0 a}^{A} & \mathbf{D}_{0 H, 0 b}^{A} & \mathbf{D}_{0 H, 0 H}^{A}
\end{array}\right|\left|\begin{array}{c}
\mathbf{U}_{2}^{A} \\
\hdashline \mathbf{U}_{0 a}^{A} \\
\mathbf{U}_{0 b}^{A} \\
\mathbf{U}_{0 H}^{A}
\end{array}\right|+\left|\begin{array}{c}
\hat{\mathbf{P}}_{2}^{A} \\
\hdashline \hat{\mathbf{P}}_{0 a}^{A} \\
\hat{\mathbf{P}}_{0 b}^{A} \\
\hat{\mathbf{P}}_{0 H}^{A}
\end{array}\right|
$$

The latter relation may be expressed in compact form for each bem-e as follows:

$\mathbf{P}_{20}^{A}=\mathbf{D}_{20,20}^{A} \mathbf{U}_{20}^{A}+\widehat{\mathbf{P}}_{20}^{A}$ for body A

$\mathbf{P}_{20}^{B}=\mathbf{D}_{20,20}^{B} \mathbf{U}_{20}^{B}+\widehat{\mathbf{P}}_{20}^{B}$ for body B

$\mathbf{P}_{0}^{C}=\mathbf{D}_{00}^{C} \mathbf{U}_{0}^{C}+\widehat{\mathbf{P}}_{0}^{C}$ for body $\mathrm{C}$

As can be noted, Eq. (28c), referring to the bem-e C, differs from Eqs. (28a) and (28b) and coincides with Eq. (14c) because it only has the interface boundary $\Gamma_{0}$.

Now the regularity conditions have to be imposed in terms of nodal compatibility

$\mathbf{U}_{0 k}=\mathbf{U}_{0 k}^{i}=\mathbf{U}_{0 k}^{j}$ or in extensive form:

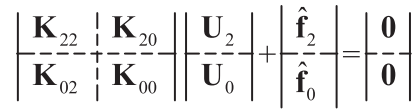

The term $\mathbf{K}_{20,20}$ is the symmetric stiffness matrix of the assembled system. It allows one to obtain the response in terms of all the nodal displacements, in a similar way to what we find in Pérez-Gavilán and Aliabadi (2001) and Kallivokas et al. (2005), in complete analogy with the FEM. Lastly, the unknown mechanical vectors $\mathbf{F}_{10}^{i}(i=\mathrm{A}, \mathrm{B}, \mathrm{C})$ are obtained by means of Eq. (23a).

The solving equation system (31) proves to be more onerous than the displacement method shown in Section 3.2 from the computational point of view. Therefore in order to reduce the unknowns, further variable condensation has to be made by working on the assembled equation system (31). In this way it is possible to remove the unknown displacements $\mathbf{U}_{0}$ of the interface nodes, thus obtaining a solving equation system having as unknowns only the nodal displacements $\mathbf{U}_{2}$ of the free boundary $\Gamma_{2}$.

Let us write Eq. (33) in the following extensive form:

$\mathbf{K}_{22} \mathbf{U}_{2}+\mathbf{K}_{20} \mathbf{U}_{0}+\widehat{\mathbf{f}}_{2}=\mathbf{0}$

$\mathbf{K}_{02} \mathbf{U}_{2}+\mathbf{K}_{00} \mathbf{U}_{0}+\widehat{\mathbf{f}}_{0}=\mathbf{0}$

If the vector $\mathbf{U}_{0}$ is deduced from Eq. (34a) and substituted in Eq (34b), the solving equation system of the displacement approach with external unknowns is obtained:

$\mathbf{H}_{22} \mathbf{U}_{2}+\widehat{\mathbf{t}}_{2}=\mathbf{0}$

where the following positions have been made:

$\mathbf{H}_{22}=\mathbf{K}_{22}-\tilde{\mathbf{K}}_{20} \mathbf{K}_{00}^{-1} \mathbf{K}_{02}$ 

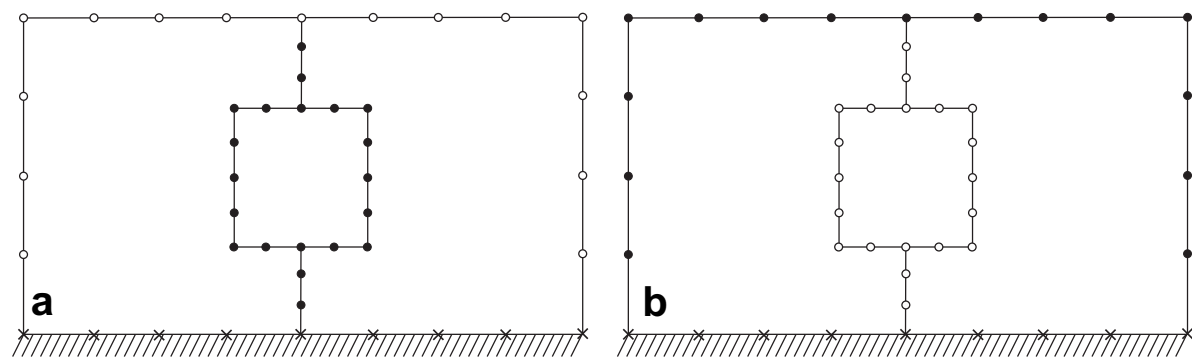

- Nodes considered in the solving system

- Nodes suppressed in the condensation process

$\times$ Constrained nodes

Fig. 4. Displacement approaches: (a) with interface variables only; and (b) with free variables only.

$\widehat{\mathbf{t}}_{2}=-\tilde{\mathbf{K}}_{20} \mathbf{K}_{00}^{-1} \widehat{\mathbf{f}}_{0}+\widehat{\mathbf{f}}_{2}$

$\mathbf{H}_{22}$ being the symmetric stiffness matrix and $\widehat{\mathbf{t}}_{2}$ the load vector, the latter defining the response on the free boundary caused by the known boundary and domain actions. Lastly, the replacement of the vector $\mathbf{U}_{2}$ in Eqs. (23a) and (34a) allows one to obtain the vectors $\mathbf{U}_{0}, \mathbf{F}_{0}$ and $\mathbf{F}_{1}$.

This method may be considered as an alternative approach to that classical one proposed by Panzeca et al. (2002b,c) and shows some advantages for multidomain analysis in Symmetric BEM analysis. The fact is that the structural analysis is carried out only considering the kinematical quantities of the external boundary and the algebraic operators show symmetry and very small dimensions. These advantages are particularly evident in the structural analysis of panels made up of stones or for plasticity problems, where the stones or the plastic cells are treated as bem-es.

Both the strategies shown in the Sections 3 and 4 have been implemented within the Karnak.sGbem (Panzeca et al., 2002a) calculus code. It is necessary to point out that both the displacement methods shown in this paper, i.e. the one based on the interface unknowns and the one with external variables, are multidomain approaches characterized by interface regularity conditions between bem-es, strong for the kinematical quantities (nodal compatibility, Eq. (15)) and weak for the mechanical ones (generalized equilibrium, Eq. (16)).

Within the SBEM formulation, an approach with mixed variables has been developed by Panzeca et al. (1999). In this latter, the regularity conditions between bem-es are assured for the kinematical and mechanical quantities both in terms of nodal variables (strong regularity) and in terms of generalized quantities (weak regularity). Obviously, the latter approach proves to be computationally more onerous than the methods shown in this paper.

\section{Summing up}

- In the displacement approach with interface unknowns (Panzeca and Salerno, 2000; Panzeca et al., 2002b,c), shown in Section 3, Eq. (17) allows one to carry out a multidomain analysis by considering as unknowns only the nodal displacements belonging to all the interface boundaries $\Gamma_{0}$ (Fig. 4a).
- In the approach, here defined as complete (Eq. (31)), the variables regard all the nodal displacements, i.e. those belonging to the interface boundaries $\Gamma_{0}$ and those belonging to the free boundaries $\Gamma_{2}$, analogously to what is done in the FEM.

- Lastly, in the displacement approach with external unknowns, shown in Section 4, Eq. (36) makes it possible for the unknowns to be only the displacements belonging to the free boundaries $\Gamma_{2}$ (Fig. 4b).

In Table 1 both the solving equation system relating to the three approaches and the additional equations required to evaluate the other unknowns in the post-processing phase are shown.

\section{Applications}

To show the effectiveness of the proposed formulation, some structures have been considered as subjected both to boundary and to domain actions. In the first two examples the results are compared to the analytical solutions (the first) and to the results obtained with the SBEM considering the body as single (the first and second) (Panzeca et al., 1998, 2001); instead, the third example shows the potentiality of the method proposed in this paper.

\subsection{Application 1}

In this application a built-in beam $(100 \times 20 \times 1 \mathrm{~cm})$, constrained on the left and loaded on the right by shearing forces having a parabolic shape with maximum value $q_{\max }=7.5 \mathrm{daN} / \mathrm{m}$ was considered.

The aim of the application is to test the model by assuming as the comparison solution both the analytical solution and the single domain solutions (Fig. 5a) obtained with the symmetric BEM. For this aim the simulation of the proposed model is made using regular and irregular meshes to show that the domain subdivision into bem-es does not substantially modify the global response of the structural system.

The following physical characteristics were assumed: Young modulus $E=10,000 \mathrm{daN} / \mathrm{cm}^{2}$, Poisson ratio $\nu=0.18$. In this simulation four different domain discretizations were utilized, i.e.:

Table 1

Summarizing table of the displacement approaches.

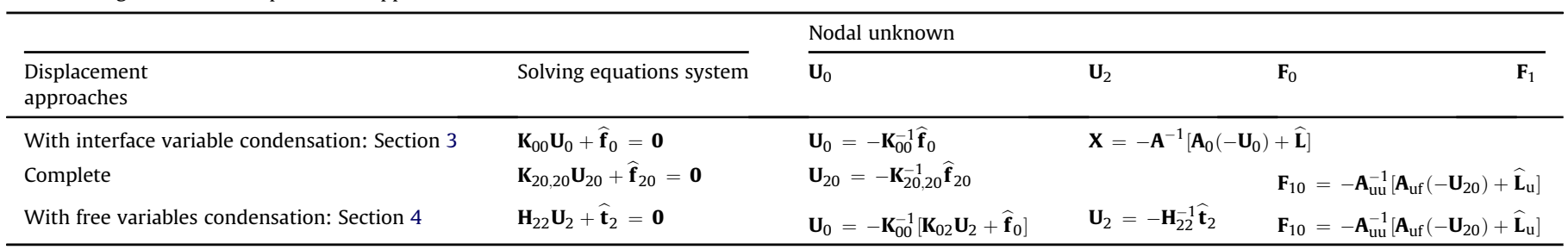



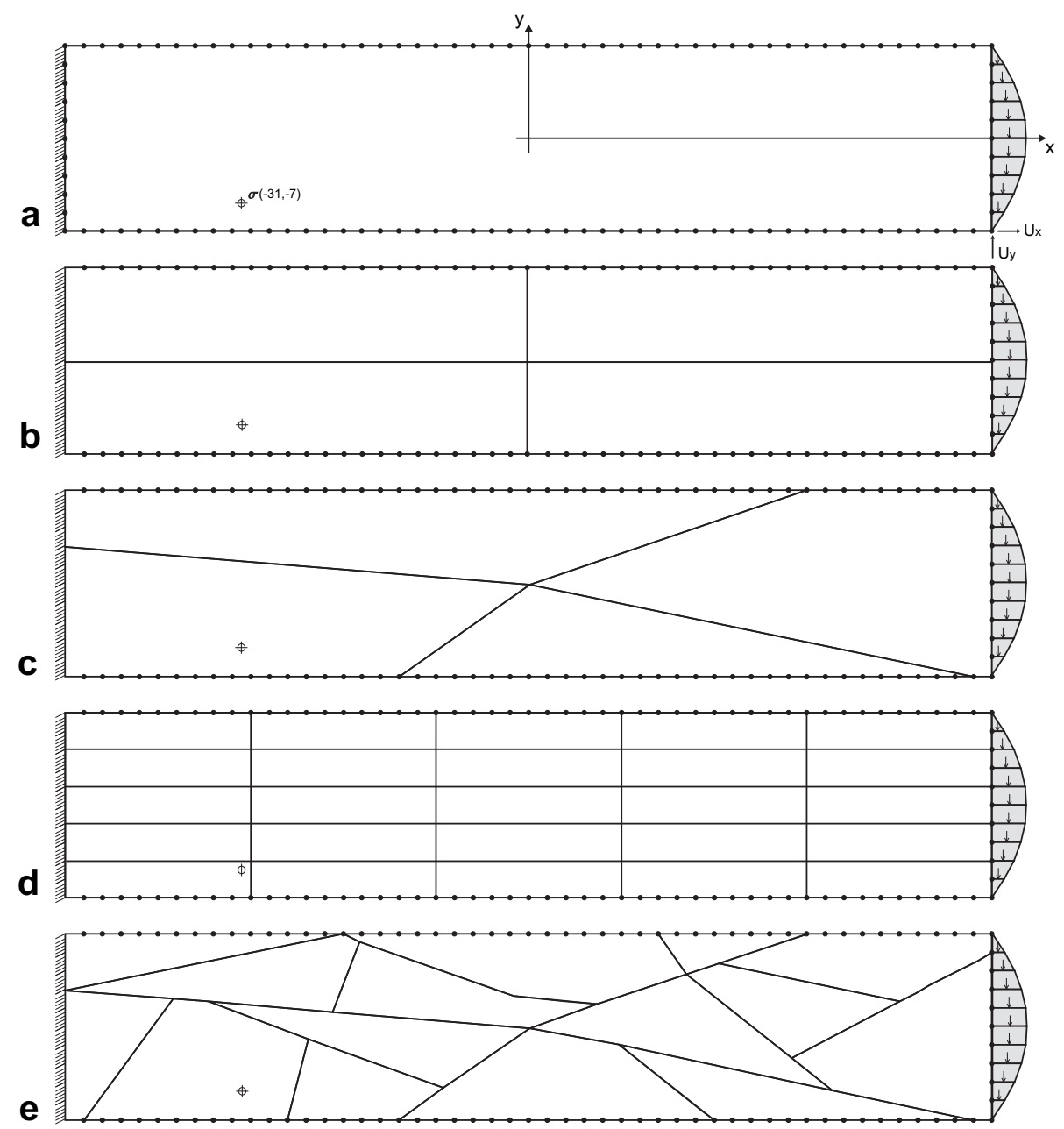

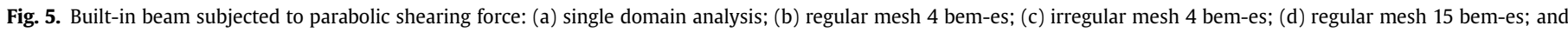
(e) irregular mesh 15 bem-es.

- Fig. 5b: regular mesh, through a domain subdivision into 4 bem-es having the same shape and dimensions;

- Fig. 5c: irregular mesh through a domain subdivision into 4 bem-es having different shapes and dimensions;

- Fig. 5d: regular mesh, through a domain subdivision into 15 bem-es having the same shape and dimensions;

- Fig. 5e: irregular mesh, through a domain subdivision into 15 bem-es having different shapes and dimensions;

In all the simulations the discretization of all the boundary types was maintained constant by using a boundary mesh step $p=2 \mathrm{~cm}$.

In Table 2 the solution in terms of nodal displacements of the low corner and of stress at the coordinate point $(-31,-7)$ is shown. As can be noted, the solution remains almost the same, showing values very close to those obtained with the analytical solution and with SBEM single domain analysis.
In Fig. 5b-e only the nodes belonging to the free boundary $\Gamma_{2}$ are shown. These nodes represent the only unknowns in the proposed formulation and define the dimensions of the algebraic operator $\mathbf{H}_{22}$ which shows the same dimensions $(218 \times 218)$ in all the fourth multidomain simulations. Instead the dimensions of the operator $\mathbf{K}_{20,20}$ are different since it provides the displacement response both of the free boundaries $\Gamma_{2}$ and of interface ones $\Gamma_{0}$ : (a) regular mesh $(332 \times 332)$, (b) irregular mesh $(364 \times 364)$, (c) regular mesh $(650 \times 650)$, $(d)$ irregular mesh $(426 \times 426)$.

\subsection{Application 2}

In this application a masonry panel, having the dimensions shown in Fig. 6a and thickness $s=40 \mathrm{~cm}$, is considered. At first the panel is thought of as a single body with homogeneous material, while subsequently it is discretized according to different strategies

Table 2

Nodal displacements and stress solution.

\begin{tabular}{|c|c|c|c|c|c|c|}
\hline Displacement stress & Analytical & Single domain (a) & Regular mesh 4 (b) & Irregular mesh 4 (c) & Regular mesh 15 (d) & Irregular mesh $15(\mathrm{e})$ \\
\hline $\mathbf{U}_{x}$ & -0.000725 & -0.000721 & -0.000719 & -0.000719 & -0.000719 & -0.000719 \\
\hline $\mathbf{U}_{y}$ & -0.004838 & -0.004917 & -0.004918 & -0.004918 & -0.004917 & -0.004917 \\
\hline$\sigma_{x}$ & -0.850500 & -0.841943 & -0.841885 & -0.841886 & -0.839739 & -0.841800 \\
\hline$\sigma_{y}$ & 0 & 0.000111 & 0.000111 & 0.000113 & 0.000137 & 0.000111 \\
\hline$\tau_{x y}$ & -0.038250 & -0.037747 & -0.037744 & -0.037740 & -0.038627 & -0.037734 \\
\hline
\end{tabular}




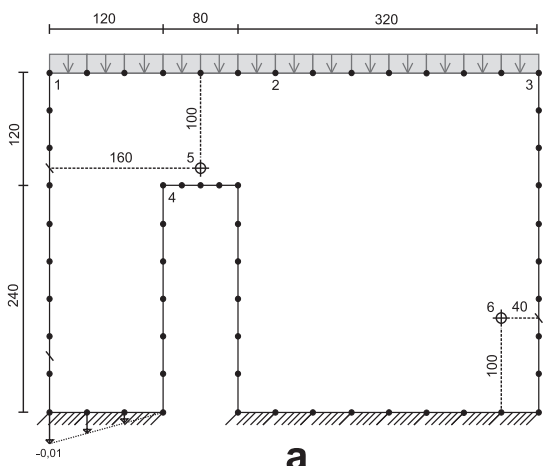

a

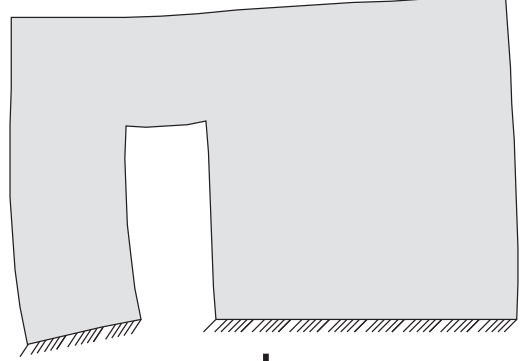

d

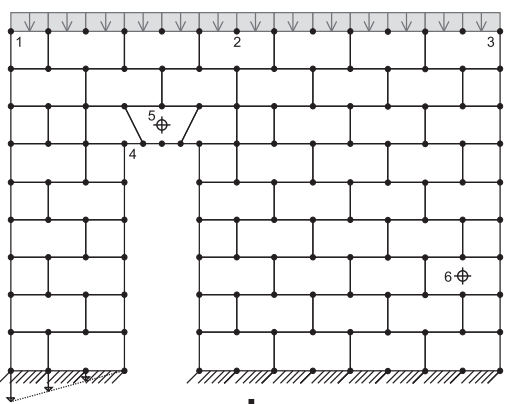

b

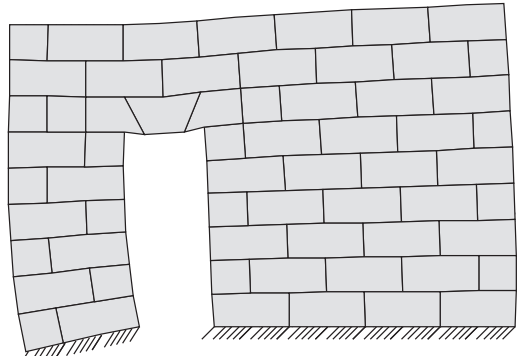

e

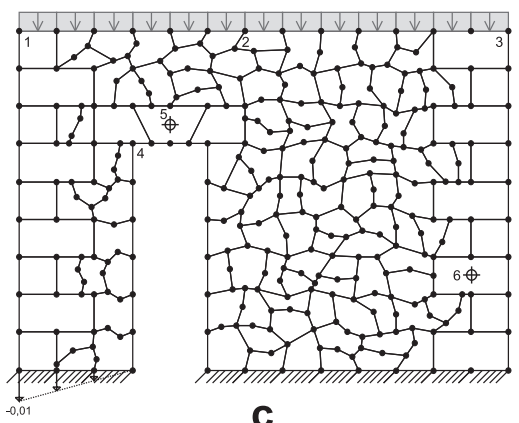

C

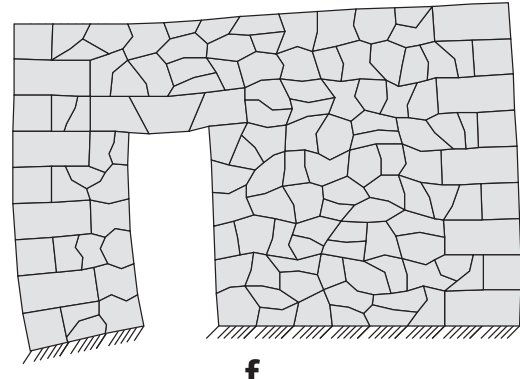

f

Fig. 6. The masonry panel: (a) single domain; (b) regular mesh; (c) irregular mesh; and (d-f) strained shapes.

(regular or irregular meshes), in the absence of mortar and gaps to make a comparison with the single-domain solution.

The panel is subjected to boundary external actions and to domain actions: a distributed load at the top $q=2000 \mathrm{daN} / \mathrm{m}$, a linear vertical displacement imposed on the left support having maximum value at the corner $u_{\max }=0.01 \mathrm{~cm}$ and the body force $\gamma=1900 \mathrm{daN} / \mathrm{m}^{3}$.

The following physical characteristics were assumed: Young modulus $E=35,000 \mathrm{daN} / \mathrm{cm}^{2}$, Poisson ratio $\nu=0.2$. In order to study this panel, two different domain discretizations were used.
- Fig. 6b: regular mesh, through a domain subdivision into 62 bem-es having the same shape and dimensions to simulate a masonry panel made up of perfectly regular stones;

- Fig. 6c: irregular mesh, through a domain subdivision into 129 bem-e having different shape and dimension to simulate a masonry panel made up of chaotic stones.

In both cases the solution was compared with the single domain solution (Fig. 5a).

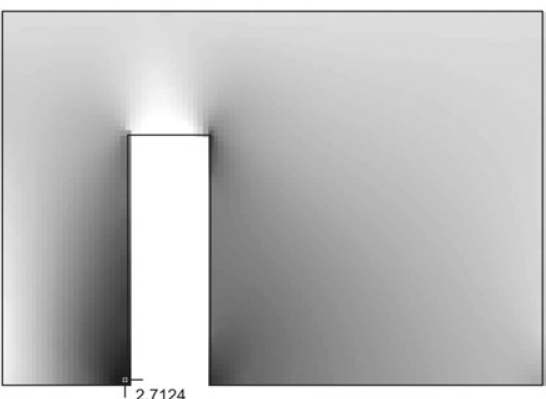

a

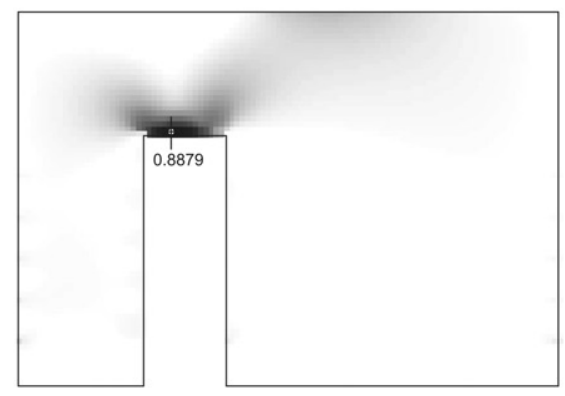

d

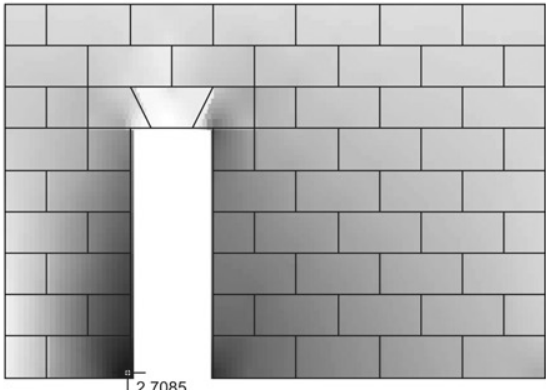

b

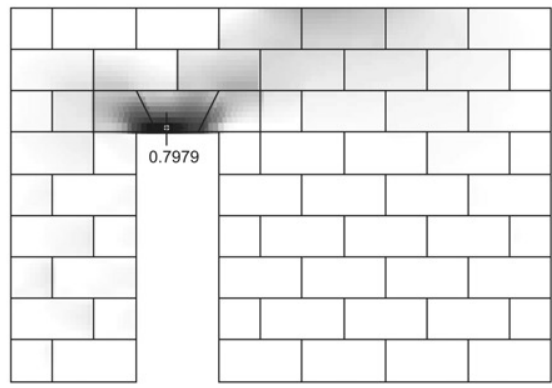

e

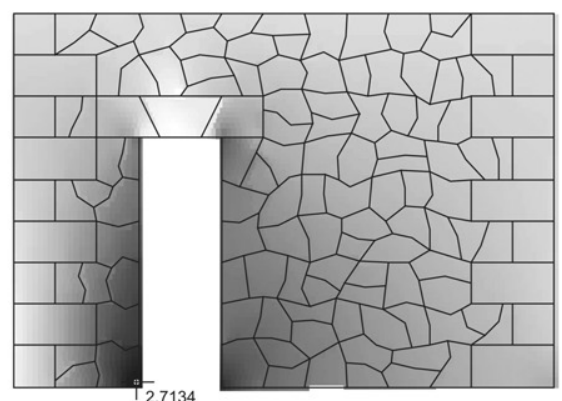

C

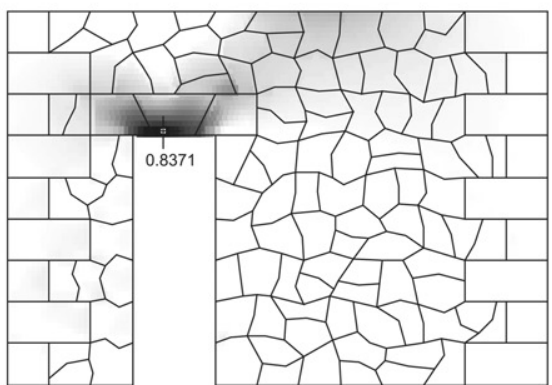

f

Fig. 7. Mapping of principal stresses: $(a-c)$ compression; and $(d-f)$ traction. 
Table 3

Nodal displacements and stress solution.

\begin{tabular}{lllll}
\hline & $\begin{array}{l}\text { Displacement, } \\
\text { stress }\end{array}$ & $\begin{array}{l}\text { Single } \\
\text { domain }\end{array}$ & $\begin{array}{l}\text { Multidomain } \\
\text { regular mesh }\end{array}$ & $\begin{array}{l}\text { Multidomain } \\
\text { irregular mesh }\end{array}$ \\
\hline 1 & $\mathbf{U}_{x}$ & -0.00626 & -0.00629 & -0.00628 \\
& $\mathbf{U}_{y}$ & -0.01438 & -0.01439 & -0.01440 \\
& $\mathbf{U}_{x}$ & -0.00662 & -0.00664 & -0.00661 \\
& $\mathbf{U}_{y}$ & -0.01174 & -0.01178 & -0.01177 \\
& $\mathbf{U}_{x}$ & -0.00440 & -0.00444 & -0.00442 \\
4 & $\mathbf{U}_{y}$ & -0.00616 & -0.00619 & -0.00620 \\
& $\mathbf{U}_{x}$ & -0.00587 & -0.00575 & -0.00575 \\
5 & $\mathbf{U}_{y}$ & -0.01282 & -0.01261 & -0.01274 \\
& $\sigma_{x}$ & 0.38565 & 0.43196 & 0.41705 \\
& $\sigma_{y}$ & -0.12839 & -0.11443 & -0.10626 \\
6 & $\tau_{x y}$ & 0.07566 & 0.08732 & 0.08398 \\
& $\sigma_{x}$ & -0.01450 & -0.01193 & -0.01351 \\
& $\sigma_{y}$ & -0.78158 & -0.78416 & -0.79431 \\
& $\tau_{x y}$ & -0.02921 & -0.02786 & -0.02811 \\
\hline
\end{tabular}

In Fig. 6d-e the three related strained shapes are shown.

The mappings of the maximum compression (Fig. 7a-c) and traction (Fig. 7d-f) principal stresses are shown. In all three cases the stress distributions are approximately the same and the maximum values are very similar and act in the same zone.

In Table 3 the solution in terms of nodal displacements $\mathbf{U}_{x}$ and $\mathbf{U}_{y}$ and of stresses $\sigma_{x}, \sigma_{y}$ and $\tau_{x y}$ evaluated at 4 boundary nodes and 2 inner points, respectively, is shown. These points are indicated in Fig. $6 a-c$.

\subsection{Application 3}

Let us consider the same masonry panel as in Section 6.2, but here considered as not homogeneous because of the presence of mortar. The global dimension and the thickness of the panel, as well as the external actions, remain the same. The physical-geometrical characteristics of the stones are: dimensions $(79 \times 39 \times 40 \mathrm{~cm})$, body force $\gamma_{\mathrm{s}}=1900 \mathrm{daN} / \mathrm{m}^{3}$, Young modulus $E_{\mathrm{s}}=35,000 \mathrm{daN} / \mathrm{cm}^{2}$, Poisson ratio $\nu_{\mathrm{s}}=0.2$. The physical-geometrical characteristics of the mortar beds are body force $\gamma_{\mathrm{m}}=1400 \mathrm{daN} / \mathrm{m}^{3}$, Young modulus $E_{\mathrm{m}}=20,000 \mathrm{daN} / \mathrm{cm}^{2}$, Poisson ratio $\nu_{\mathrm{m}}=0.2$.

For this simulation two different domain discretizations were utilized.

- Fig. 8a and c: regular mesh, through a domain subdivision into 124 bem-es, i.e. 62 bem-es to simulate the regular stones and 102 bem-es to simulate the presence of horizontal and vertical mortar beds both having the same width $s=1 \mathrm{~cm}$.

- Fig. 8d and f: irregular mesh, through a domain subdivision into 258 bem-es, i.e. 201 bem-es to simulate the chaotic unformed stones and 57 bem-es to simulate the presence of the mortar having a width varying between 1 and $3 \mathrm{~cm}$.

In Fig. 8a and $d$ the domain mesh discretization is shown, whereas in Fig. 8b and e two details of the stone-mortar connection are represented and in Fig. $8 \mathrm{c}$ and $\mathrm{f}$ the two strained shapes.

In Fig. 9a, b, d, and e the mappings of principal traction and compression stresses in the stones only are shown, where it can be noted that the presence of the mortar does not substantially modify the inner distribution of the stresses and the related maximum values. In Fig. 9c and $f$ the distributions of the normal stresses are shown along the horizontal and vertical sections, each computed at 70 points.

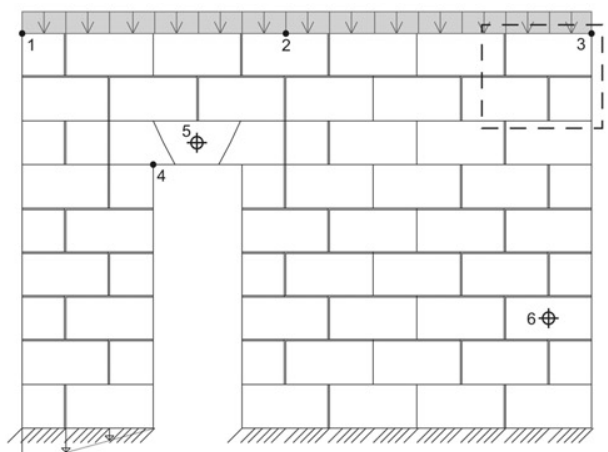

a

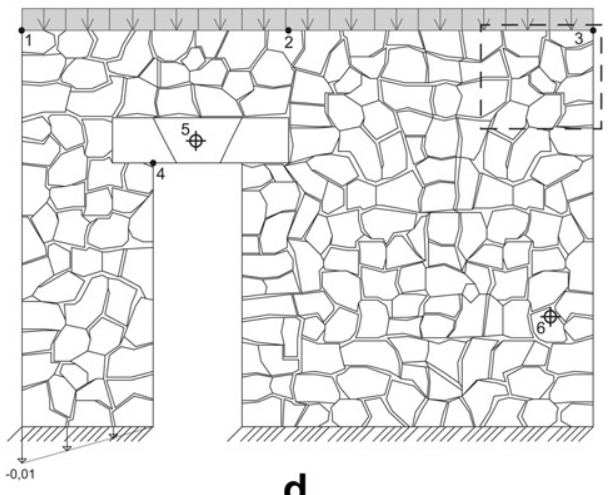

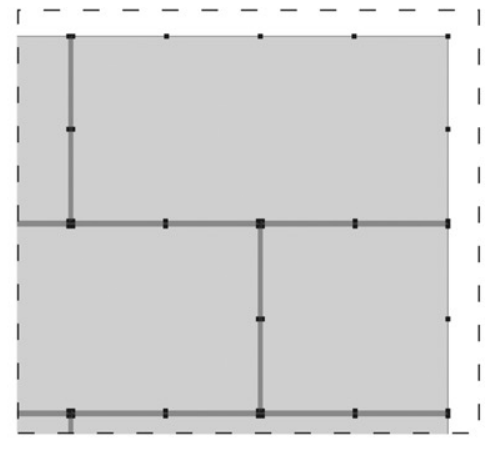

b

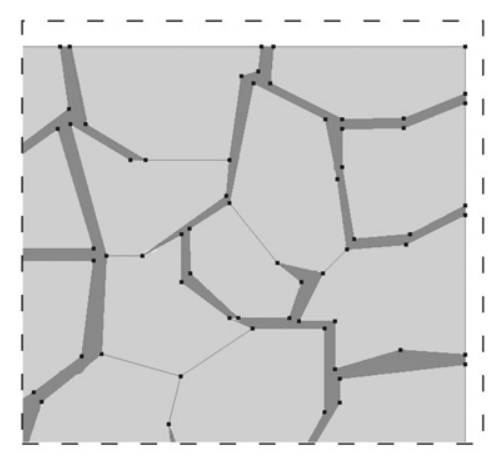

e

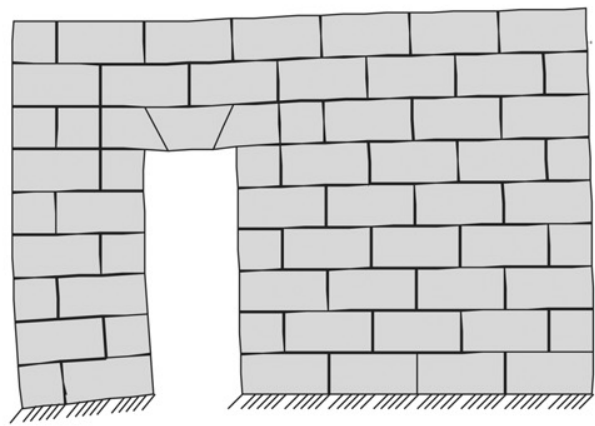

C

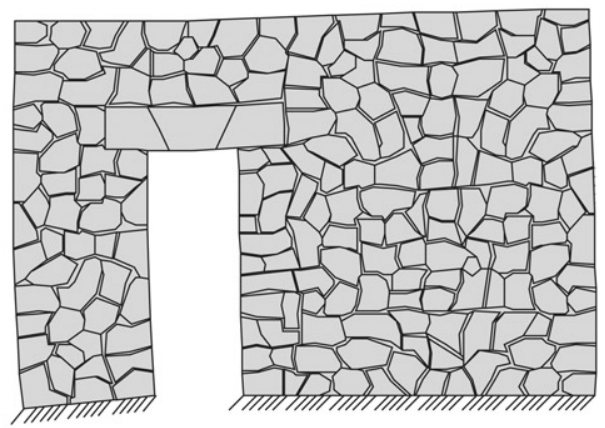

f

Fig. 8. The masonry panel: (a) regular and (d) irregular meshes; (b), (e) some details of stone-mortar connection; and (c, f) strained shapes. 


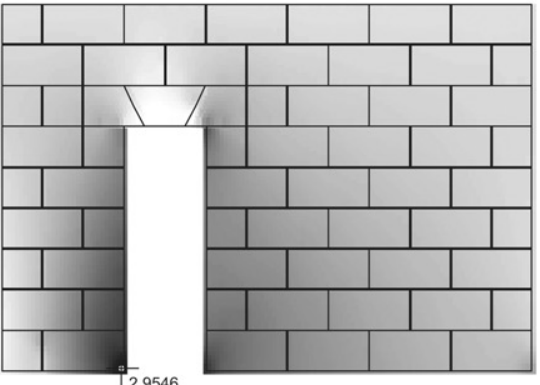

a

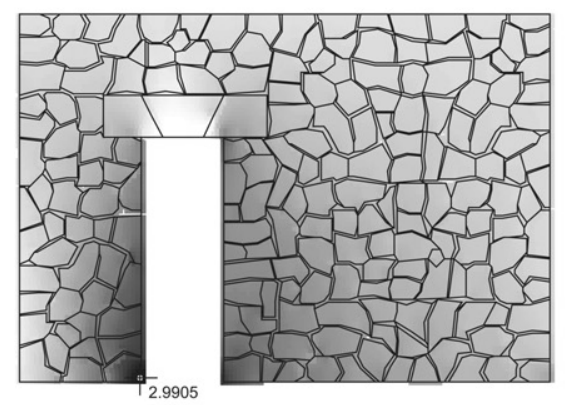

d

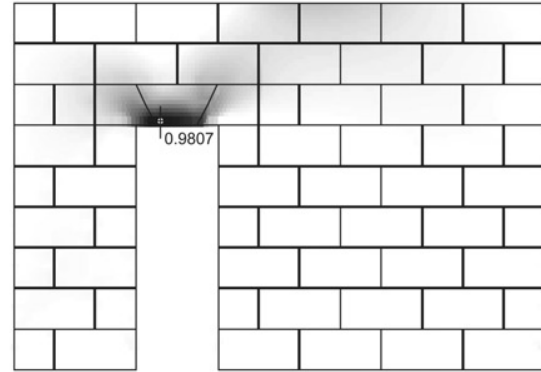

b

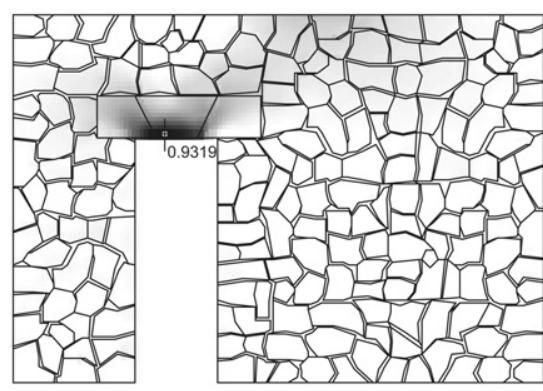

e

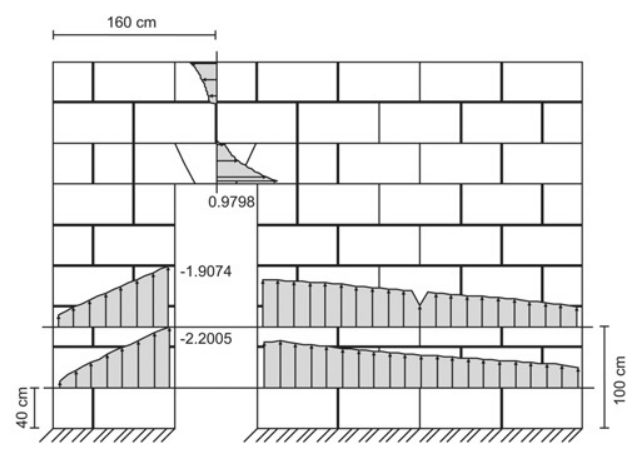

C

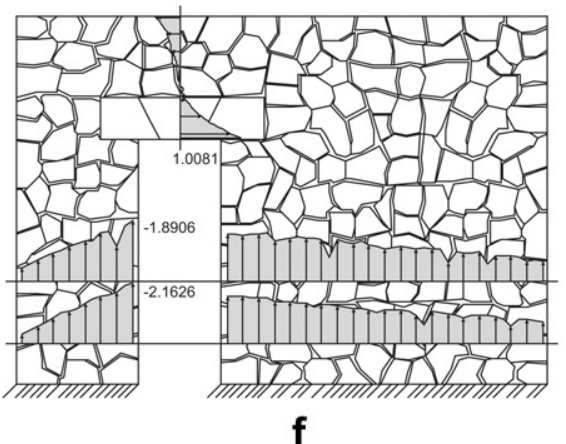

Fig. 9. Stress distributions: (a, d) compression stress and (b, e) traction stress mappings; and (c, f) normal stress distribution along horizontal sections.

In the vertical section made in the middle of the door opening a different normal stress function may be noted. Indeed in Fig. 9c the section passes through the vertical mortar layers and the related normal stress value shows a very low value compared with those computed in the low and upper stones. By contrast, in Fig. 9f the function appears to be continuous.

In the horizontal distributions related to vertical stresses there are some abrupt jumps. This is due to the position of the point where the stress is computed, i.e. in the stone or in the mortar.

In Table 4 the solution in terms of nodal displacements $\mathbf{U}_{x}$ and $\mathbf{U}_{y}$ and of stresses $\sigma_{x}, \sigma_{y}$ and $\tau_{x y}$ evaluated at 4 boundary nodes and 2 inner points, respectively, is shown. These points are indicated in Fig. 8a and d.

Table 5 shows the CPU times required using the different displacement approaches. These tests were performed on a personal computer equipped with a $2.5 \mathrm{GHz}$ Core2Duo processor and 8 GB of Ram.

Table 4

Nodal displacements and of stress solution.

\begin{tabular}{clll}
\hline & Displacement, stress & Regular mesh & Irregular mesh \\
\hline \multirow{3}{*}{2} & $\mathbf{U}_{x}$ & -0.00622 & -0.00635 \\
& $\mathbf{U}_{y}$ & -0.01450 & -0.01513 \\
& $\mathbf{U}_{x}$ & -0.00662 & -0.00668 \\
& $\mathbf{U}_{y}$ & -0.01194 & -0.01201 \\
& $\mathbf{U}_{x}$ & -0.00440 & -0.00447 \\
& $\mathbf{U}_{y}$ & -0.00630 & -0.00672 \\
& $\mathbf{U}_{x}$ & -0.00577 & -0.00576 \\
& $\mathbf{U}_{y}$ & -0.01283 & -0.01345 \\
& $\sigma_{x}$ & 0.39412 & 0.38735 \\
& $\sigma_{y}$ & -0.03166 & -0.04368 \\
& $\tau_{x y}$ & 0.07775 & 0.08021 \\
& $\sigma_{x}$ & -0.011394 & -0.01426 \\
& $\sigma_{y}$ & -0.77423 & -0.79201 \\
& $\tau_{x y}$ & -0.02766 & -0.03001 \\
\hline
\end{tabular}

Table 5

CPU times with different displacement approaches.

\begin{tabular}{clllc}
\hline & $\begin{array}{l}\text { Displacement } \\
\text { approaches }\end{array}$ & $\begin{array}{l}\text { Nodal } \\
\text { interface } \\
\text { nodes }\end{array}$ & $\begin{array}{l}\text { Nodal free } \\
\text { nodes }\end{array}$ & CPU times [s] \\
\hline $\begin{array}{c}\text { Regular } \\
\text { mesh }\end{array}$ & With interface variables & 1474 & 52 & 82.5 \\
$\begin{array}{c}\text { Irregular } \\
\text { mesh }\end{array}$ & With interface variables & 2901 & 61 & 63.8 \\
\hline
\end{tabular}

\section{Conclusions}

Within the Boundary Element Method applied to in-plane loaded structural systems, the symmetric formulation is of considerable significance, mainly as regards the computational aspects. In this formulation, a sub-structuring makes it possible to obtain some results for bodies having zone-wise physical and geometrical characteristics.

The approach proposed in this paper employs sub-structures called, as in the FEM, bem-elements having large or small dimensions, inside which the equilibrium and compatibility conditions are satisfied at every point because the fundamental solutions are used.

A displacement approach with free variables in which the main variables are only the nodal displacements of the free boundary of all the assembled system has been shown. This approach is a natural evolution of the classical displacement approach with interface variables developed by Panzeca and Salerno (2000) and Panzeca et al. (2002b,c); this choice arises from the need to reduce the computational burden when the mesh proves to be very dense as in the case of elastoplastic analysis through step-by-step strategies.

For each bem-e a relationship between generalized tractions, nodal displacements and external actions, all quantities defined both on the interface between adjacent bem-es and on the free 
boundaries, is evaluated. The regularity conditions written at the interfaces between the bem-es and a subsequent condensation process lead to a solving equation system having reduced dimensions due to the presence of only the nodal displacements of the free boundary.

This strategy has been implemented inside the Karnak.sGbem calculus code (Panzeca et al., 2002a) and some applications show the effectiveness of the response compared to the analytical solutions, when the latter are applicable, or to the single domain formulation with the symmetric BEM.

\section{References}

Aliabadi, M.H., 2002. The Boundary Element Method. John Wiley \& Sons, New York. Bonnet, M., 1995. Regularized direct and indirect symmetric variational BIE formulation for three-dimensional elasticity. Eng. Anal. Boundary Elem. 15, 93-102.

Cen, Z., Chen, T., Wang, B., Wu, Z., 1999. A symmetric Galerkin multi-zone boundary element method for cohesive crack growth. Eng. Fract. Mech. 63, 591-609.

Cook, R.D., 1989. Concepts and Applications of Finite Element Analysis. John Wiley \& Sons, New York.

Frangi, A., Novati, G., 1996. Symmetric BE method in two-dimensional elasticity: evaluation of double integrals for curved elements. Comput. Mech. 19, 58-68.

Ganguly, S., Layton, J.B., Balakrishna, C., Kane, J.H., 1999. A fully symmetric multizone Galerkin boundary element method. Int. J. Numer. Methods Eng. 44, 991-1009.

Gray, L.J., Martha, L.F., Ingraffea, A.R., 1990. Hypersingular integrals in boundary element fracture analysis. Int. J. Numer. Methods Eng. 29, 1135-1158.

Gray, L.J., Paulino, G.H., 1997. Symmetric Galerkin boundary integral formulation for interface and multi-zone problems. Int. J. Numer. Methods Eng. 40, 3085-3101.

Guiggiani, M., 1995. Hypersingular boundary integral equation have an additional free term. Comput. Mech. 16, 245-248.

Holzer, S., 1993. How to deal with hypersingular integrals in the symmetric BEM. Commun. Numer. Methods Eng. 9, 219-232.

Kallivokas, L.F., Juneja, T., Bielak, J., 2005. A symmetric Galerkin BEM variational framework for multi-domain interface problems. Comput. Meth. Appl. Mech. Eng. 194, 3607-3636.

Layton, J.B., Ganguly, S., Balakrishna, C., Kane, J.H., 1997. A symmetric Galerkin multi-zone boundary element formulation. Int. J. Numer. Methods. Eng. 40, 2913-2931.

Maier, G., Polizzotto, C., 1987. A Galerkin approach to boundary element elastoplastic analysis. Comput. Meth. Appl. Mech. Eng. 60, 175-194.
Maier, G., Diligenti, M., Carini, A., 1991. A variational approach to boundary element elastodynamic analysis and extension to multidomain problems. Comput. Meth. Appl. Mech. Eng. 92, 192-213.

Panzeca, T., Fujita Yashima, H., Salerno, M., 1998. Mathematical aspects and applications of the symmetric Galerkin boundary element method. In: Idelsohn, S.R. Onate, E., Dvorkin, E.N. (Eds.), Fourth World Congress on Computational Mechanics (WCCM), CDROM. Buones Aires, Argentina.

Panzeca T, Salerno M., Terravecchia S., 1999. Subeg in the symmetric symmetric BEM. In: XIV AIMETA Congress, Como, Italy, CD-ROM.

Panzeca, T., Salerno, M., 2000. Macro-elements in the mixed boundary value problems. Comput. Mech. 26, 437-446.

Panzeca, T., Fujita Yashima, H., Salerno, M., 2001. Direct stiffness matrices of BEs in the Galerkin BEM formulation. Eur. J. Mech. A/Solids 20, 277-298.

Panzeca, T., Cucco, F., Terravecchia, S., 2002a. Karnak.sGbem. Release 1.0. www. bemsoft.it Palermo.

Panzeca, T., Cucco, F., Terravecchia, S., 2002b. Symmetric boundary element method versus finite element method. Comput. Meth. Appl. Mech. Eng. 191, 3347-3367.

Panzeca, T., Salerno, M., Terravecchia, S., 2002c. Domain decomposition in the symmetric boundary element method analysis. Comput. Mech. 28, 191-201.

Panzeca, T., Cucco, F., Milana, V., Terravecchia, S., 2004. Multidomain approach for thermoelasticity in the SGBEM. In: Fifth International Conference on Boundary Element Techniques, Beteq 2004, Madeira, Portugal.

Panzeca, T., Salerno, M., Terravecchia, S., Zito, L, 2008. The symmetric boundary element method for unilateral contact problems. Comput. Meth. Appl. Mech. 197, 2667-2679.

Panzeca, T., Zito, L., Terravecchia, S., 2009. Internal spring distribution for quasi brittle fracture via symmetric boundary element method. Eur. J. Mech. A/Solids 28, 354-367.

Pérez-Gavilán, J.J., Aliabadi, M.H., 2001. A symmetric Galerkin Bem for multi-connected bodies: a new approach. Eng. Anal. Boundary Elem. 25, 633-638.

Polizzotto, C., 1988a. An energy approach to the boundary element method, part. I: Elastic solids. Comput. Meth. Appl. Mech. Eng. 69, 167-184.

Polizzotto, C., 1988b. An energy approach to the boundary element method, part. II: elastic-plastic solids. Comput. Meth. Appl. Mech. Eng. 69, 263-276.

Polizzotto, C., 1991. A boundary min-max principle as a tool for boundary element formulation. Eng. Anal. Boundary Elem. 2, 89-93.

Portela, A., Aliabadi, M.H., Rooke, D.P., 1992. Dual boundary element method: effective implementation for crack problem. Int. J. Numer. Methods Eng. 33, 1269-1287.

Salvadori, A., 2001. Analytical integration of hypersingular kernel in 3D BEM problems. Comput. Meth. Appl. Mech. Eng. 190, 3957-3975.

Terravecchia, S., 2006. Closed form coefficients in the symmetric boundary element approach. Eng. Anal. Boundary Elem. Meth. 30, 479-488.

Vodička, R., Mantič, V., París, F., 2007. Symmetric variational formulation of bie for domain decomposition problems in elasticity - an SGBEM approach for nonconforming discretizations of curved interfaces. Comput. Model. Eng. Sci. 4, 31-50. 